\title{
Raffaello e Omero (da Urbino alla Stanza della Segnatura)*
}

La ricerca del volto perduto di Omero è stata perseguita dalla più remota antichità all'età contemporanea e non solo nel mondo occidentale. Uno dei più recenti ritratti di Omero è l'opera Homer, realizzata nel 2014 da un artista sud coreano, Joongwon Jeong: ${ }^{1}$ una gigantografia iperrealistica, in cui ogni tratto del volto del Poeta, ruga, capello, ogni piega della minima porzione dell'abito rappresentato è resa con esasperato e minuzioso realismo. Dell'atto creativo di Jeong interessano qui soprattutto la scelta stilistica ed estetica e la ricerca del modello. Attraverso lo stile e l'estetica iperrealistici il volto di Omero assume le sembianze di un volto fotografato, le quali provocano, in chi guarda l'opera, l'illusione che quel volto, dunque Omero, sia realmente esistito, e in un tempo pressoché contemporaneo a quello della fruizione dell'opera, e inducono persino a ritenere che il Poeta possa essere in vita nel momento in cui ne viene osservato il ritratto. Quel volto, per essere più che realistico, è paradossalmente anche ideale: negli occhi, ad esempio, la mimesi marcatamente reale della cecità ${ }^{2}$ giunge a rappresentare l'idea stessa della cecità.

L'assenza della vista, che in un poeta è simbolo di ispirato vedere poetico e straordinaria capacità mnemonica, appartiene più e prima di ogni vate a Omero, al punto che quasi nessuno dei suoi ritratti, fin dal più antico, l'ha taciuta. ${ }^{3}$ La cecità è dunque il fil ronge, l'elemento archetipico e ineludibile dei ritratti omerici con cui si sono misurati gli artisti. L'opera contemporanea Homer si ispira principalmente a una copia del noto Omero Farnese, ${ }^{4}$ contaminata con modelli pittorici ${ }^{5}$ e, soprattutto, con l'immagine fotografica di un

* Desidero ringraziare Concetta Bianco, Gerardo Guccini, Nicoletta Guidobaldi, Joongwon Jeong, Vincenzo Trione, Ranieri Varese.

1 https://www.deviantart.com/jw-jeong; https://blog.naver.com/carandini; immagini dell'opera Homer, acrilico su tela $(112 \times 162 \mathrm{~cm}$.): https://blog.naver.com/carandini/40190963900.

2 Joongwon Jeong ha dipinto la cecità di Omero come una 〈reale` cataratta, «my artistic licence», ha dichiarato a una nostra domanda.

${ }^{3}$ Del più antico ritratto di Omero, realizzato nella metà del V sec. a.C., sono conservate solo copie, tra le quali una, particolareggiata, della prima età imperiale (marmo, h. 39, 7 cm. München, Glypothek, 273). Fin dall'antichità, costituiscono una vera e propria eccezione le immagini di Omero vedente; fra le pochissime, per epoche relativamente vicine all'Omero di Raffaello, ricordo il peculiare ritratto di «Homero poeta sovrano», eseguito da Giovanni Boccaccio (1360 circa), forse anche come sua ‘maschera`, per il quale rinvio all'ottima analisi di Ricci Battaglia 2018, 35-41.

${ }^{4}$ L'Omero della Collezione Farnese è una replica d'età antonina di un ritratto di Omero tardo ellenistico (I sec. a.C. Marmo, Napoli, Museo Nazionale Archeologico di Napoli. https://www.museoarcheologiconapoli.it/it/le-collezioni/collezione-farnese-2/ritratti/).

5 Joongwon Jeong ha segnalato queste come fonti di ispirazione: Homère et son guide (Homer and his Guide) di William Adolphe Bouguereau (1874. Olio su tela; 2,08 × 1,42 m. Milwakee, Milwakee Art Museum, M1963.134. http://collection.mam.org/details.php?id=14910); Homère déifié (L'Apothéose d'Homère) di Jean-Auguste-Dominique Ingres (1827. Olio su tela; 3,86 × 5,12 m. Paris, Musée du Louvre, 5417. https:// www.louvre.fr/oeuvre-notices/homere-deifie). 
uomo il cui volto reale è stato considerato da Jeong idealmente evocativo dei volti ricostruiti di Omero. ${ }^{6}$

Ferme restando le differenze stilistiche, estetiche, storiche, culturali, Jeong ha compiuto un'operazione già attuata da artisti ellenistici, che hanno cercato nel marmo il volto di Omero, e da quelli che lo hanno realizzato in età imperiale, copiando opere di II e I sec. a.C.: accentuare realisticamente i tratti di un volto simulato, un volto non reale ma che si può solo immaginare. ${ }^{7}$ Dai più antichi ritratti di Omero ai più recenti, tutti sono l'esito di una ricostruzione che molto deve all'immaginario, formatosi nel tempo attraverso la stratificazione di suggestioni letterarie (dall'Iliade e dall'Odissea, dalle Vite di Omero, dalla tradizione indiretta sul Poeta, e da altro) e iconografiche.

Anche se la ricerca della maggior parte degli artisti si è concentrata sul volto di Omero, non pochi di essi hanno voluto dare al Poeta anche un corpo: tra questi, Raffaello ${ }^{8}$ che, nel suo Omero del Parnaso vaticano, ha creato una immagine-summa del Poeta e una figura volta a un dialogo multiplo. Lo scopo di questo articolo è indagare e interrogare l'Omero dell'artista urbinate sia in sé stesso sia nelle relazioni iconografiche e di senso che intercorrono tra l'immagine del Poeta e altre figure del Parnaso. La Stanza della Segnatura, che accoglie l'affresco, era aperta sul Cortile del Belvedere che, proprio a ridosso dell'inizio dei lavori di Raffaello, ospitava una delle più importanti scoperte archeologiche del Rinascimento: il gruppo scultoreo del Laocoonte. L'asse iconografico e iconologico tra quest'opera, Omero e il giovane che, seduto alla sua destra, accoglie le parole del Poeta costituisce un punto chiave della nostra ricerca, come pure è nostro oggetto di interesse la ricostruzione degli eterogenei stimoli culturali e letterari che intervennero nella ricezione di Omero nella Roma di Papa Giulio II. ${ }^{9}$

${ }^{6}$ «Since I could not rely solely on my imagination to come up with the lifelike image of the ancient poet», ha dichiarato Jeong, "I found a model who had a similar facial feature with the bust of Homer. And then I combined the image of his real face with the face of the marble bust. The general facial structure was strictly based on the bust. Everything else that makes Homer life-like, such as the aged skin, the moles and colours of the eyes and hair, were all taken from the model's real face», https://www.thestar.com.my/lifestyle/ culture/2014/08/08/joongwon-jeong-hyperrealist-artist.

${ }^{7}$ Jeong, che è partito non a caso da un ritratto ellenistico (pur nella versione della sua copia d'età antonina), si è spinto concettualmente oltre. Ha infatti creato un ritratto di Omero che è un dialogo a distanza fra simulazioni, per giunta distanti nel tempo: quella cronologicamente più recente è la più paradossale e ossimorica, essendo la fotografia di un volto reale. Dunque l'ultimo - al momento - ritratto di Omero è un volto in cui si incontrano ricostruzione, simulazione, realtà: un volto tanto reale quanto simulato che più che ricostruire le sembianze di Omero ricostruisce un'idea di Omero e ingenera, intenzionalmente, nell'osservatore dell'opera smarrimento, ad un tempo poetico e reale.

8 Prima di lui, ad esempio, lo scultore ellenistico Archelaos di Priene nel marmoreo rilievo votivo di un poeta con raffigurazione dell'apoteosi di Omero (London, British Museum 1819,0812.1. https://www. britishmuseum.org/collection/object/G_1819-0812-1); dopo Raffaello, ad esempio, Sir Thomas Lawrence, Homer reciting his Poems (1790. Olio su tela, $94 \times 111,1 \mathrm{~cm}$.; London, Tate Britain T01974. https://www. tate.org.uk/art/artworks/lawrence-homer-reciting-his-poems-t01974).

9 Questo articolo si inserisce all'interno di una più ampia ricerca sull'Omero di Raffaello e su altri poeti del Parnaso, svolta da Sotera Fornaro e da chi scrive, di cui si darà conto in un libro in preparazione. Sullo specifico tema dell'Omero vaticano si vedano inoltre: Fornaro 2018, 1025-1042; Fornaro/Viccei 2020, 17-41, anche per altra bibliografia su Raffaello, comprensibilmente sterminata. In questo articolo, vengono citati gli studi che più di altri sono stati essenziali per il tema specifico affrontato e si è tenuto conto di puntuali panoramiche bibliografiche, anche ragionate, come: Shearman 2003, 1537-1635; Farinella 2004, 111-125; Chapman/Henry/Plazzotta 2005, 307-315; Di Teodoro/Farinella 2017, 431-435. 


\section{Stratigrafia di una immagine. Homerus Smyrnaeus nello Studiolo del Palazzo Ducale di Urbino e il Tempietto delle Muse.}

Nell'immaginario artistico di Raffaello, il volto di Omero si è manifestato precocemente. Anche se è impossibile determinare con precisione quando ciò sia avvenuto, è verosimile pensare a date comprese tra la fine dell'infanzia e l'adolescenza, mentre si possono indicare con certezza i luoghi di questi incontri: lo Studiolo del Palazzo Ducale di Urbino e il Tempietto delle Muse, cui lavorò il padre di Raffaello, Giovanni Santi.

Non può dunque iniziare che da Urbino, una delle città simbolo del Rinascimento italiano, del mecenatismo artistico e letterario, crocevia di fondamentali elaborazioni culturali, urbanistiche, architettoniche, pittoriche e luogo cruciale per la prima formazione di Raffaello, ${ }^{10}$ la complessa ricerca delle radici del suo Omero del Parnaso vaticano, dove l'Ur-poeta viene inserito in una rete di relazioni, all'interno delle quali risalta il collegamento con Apollo Musagete.

Nello Studiolo del Duca di Montefeltro (1472-1476), ${ }^{11}$ «sintesi didattico-enciclopedica del cuore della cultura rinascimentale», ${ }^{12}$ luogo per la meditazione intellettuale e spazio autocelebrativo all'interno di un Palazzo che era un manifesto del potere e della cultura di corte,,$^{13}$ i ritratti di ventotto Dotti (o Uomini Illustri) raccontano, dalla più remota antichità al Quattrocento, il quid proprium. Fra tutti, il primo in ordine di tempo è Omero, dipinto nel registro superiore della parete orientale, tra Virgilio e Seneca: secondo la inveterata tradizione, il Poeta è cieco; sul suo capo è una corona d'alloro. HOMERO SMYR$N A E O$, cujus poësin ob divinam disciplinarum varietatem omnis aetas admirata est, assecutus nemo post, gratitudo pos[uit]: ${ }^{14}$ al di sotto del ritratto, il Duca e quanti erano ammessi nel suo Studiolo leggevano queste parole encomiastiche. La mano sinistra di Omero è poggiata su un libro che, nel caso suo e degli altri Dotti, è simbolo del lavoro intellettuale e che rinvia, al contempo e in senso lato, alla bibliofilia di Federico, «amatore del libro bello e ricco, da sfogliare più che da leggere, oggetto prezioso da collezione più che strumento di studio». ${ }^{15}$

${ }^{10}$ Urbino, con il suo Palazzo Ducale, ha giocato un ruolo molto importante nella genesi e nelle prime elaborazioni della immensa ed eccezionale cultura figurativa di Raffaello, ugualmente il padre Giovanni Santi, artista dal multiforme ingegno - pittore, poeta, regista e scenografo di corte (tra i più noti spettacoli: Amore al tribunale della Pudicizia, 1474, in onore di Federico d'Aragona) - e, dopo la morte, la sua bottega. Su questi temi: Michelini Tocci 1985; Iacobini 1999, 198-205; Varese 1994; Id. 1999; Id. 2018, 75-79; Henry/ Mancini 2006; Mochi Onori 2009; Butler 2009, 38-43; Rodeschini/Daffra/Di Pietrantonio 2018, 15-29, 129-159; Rotondi Terminiello 2018, 15-18; Valazzi 2018.

${ }_{11}$ Sullo Studiolo del Duca Federico da Montefeltro, opera dei pittori Giusto di Gand e Pedro Berruguete, Marchi 2015. Per un'analisi storica, culturale, tipologica dello Studiolo, Cieri Via 2005.

12 Valazzi 2015, 14.

${ }^{13}$ Frommel 2004, 167-196. A rendere tale il Palazzo dei Montefeltro, contribuì in modo sostanziale la stessa ricchissima biblioteca, Peruzzi 2014, in particolare, 337-354.

14 «A Omero di Smirne, la cui poesia, per la divina varietà dei suoi insegnamenti è stata ammirata in ogni età, e mai eguagliata, la gratitudine pose», Marchi 2015, 148, VII, per la traduzione e l'intera epigrafe, oggi leggibile solo limitatamente al dativo onomastico Homero Smirnaeo. Moormann 2004, 134-135.

15 Michelini Tocci 1985, 12. Questo non osta alla intensa, voluta e mirata politica culturale di Federico (Puddu 1986, 488), della quale la biblioteca era tassello fondamentale, e per la quale fu essenziale la figura di Ottaviano Ubaldini della Carda, Bertuzzi 2018, 146-169, anche per la precedente bibliografia. 
Nello Studiolo, Omero è presente anche attraverso riflessi della sua poesia epica che si colgono implicitamente nel Dotto Bessarione, identificabile grazie all'epigrafe onomastica. ${ }^{16}$ Il cardinale e umanista, che aveva un legame stretto e preferenziale con Federico, al quale diede in custodia la sua pregevolissima biblioteca, e con i suoi figli, donò proprio a uno di essi, Antonio, un codice greco con l'Iliade (Urb. Gr. 137). ${ }^{17} \mathrm{E}$ infine, ancor più sottilmente, nel ritratto di Pio II, pontefice ma anche mecenate, umanista, scrittore, conoscitore del greco (era stato allievo di Francesco Filelfo) che, in un incontro con Federico a Tivoli (1461), esaltò Omero e l'Iliade soprattutto per la descrizione delle armi, l'importanza della guerra di Troia e per la fama di eroi quali Achille, Agamennone, Ettore. ${ }^{18}$

A Raffaello non era precluso l'accesso a Palazzo Ducale, certo fino a quando fu in vita suo padre (1494), ${ }^{19}$ uno dei pittori di corte più apprezzati e poeta, famoso soprattutto per il poema epico celebrativo La vita e le gesta di Federico di Montefeltro duca d'Urbino. ${ }^{20}$ Raffaello conosceva lo Studiolo, come dimostrano anche copie di alcuni Dotti nel Libretto veneziano e - ciò massimamente ci interessa - la copia puntuale di Omero, accompagnata da uno schizzo del solo volto. ${ }^{21}$ Non di meno doveva essere noto a Raffaello il Tempietto delle Muse (1476-1480) che, insieme alla coeva Cappella del Perdono, era stato costruito in relazione al soprastante Studiolo. ${ }^{22}$ «Nell'ambito di un programma organico, che legava idealmente e strutturalmente gli ambienti riservati al Duca nell'area occidentale del Palazzo [...], la cappella e il Tempietto costituiscono, idealmente, il punto d'arrivo del

${ }^{16}$ Collocato nella parete meridionale, tra Pio II e Alberto Magno, il ritratto è conservato a Parigi (Musée du Louvre). Il nome al dativo (BESSARIONI) è dipinto al di sotto dell'immagine, per la quale, corredata da una breve scheda, rinvio a http://www.regione.marche.it/Regione-Utile/Cultura/Catalogo-beni-culturali/ RicercaCatalogoBeni/ids/53773/Ritratto-del-cardinale-Bessarione.

17 Sulla figura di Bessarione, morto lo stesso anno in cui si data l'inizio dei lavori dello Studiolo (1472), più specificatamente sui rapporti del cardinale e del circolo bessarioneo con Urbino e Federico in particolare, rinvio agli studi di Concetta Bianco e alla ricchissima bibliografia lì citata: Bianco 1986, 61-79, in particolare 63-64; Ead. 1999; Ead. 2004, 127-145. Sulla presenza di Omero e della sua Iliade nella biblioteca urbinate, Peruzzi 2014, 348.

18 Stok 2018, 169.

19 Raffaello dovette accedere ancora al Palazzo negli anni immediatamente successivi la morte di Giovanni Santi, quando lavorava, pur in modo saltuario, nella bottega del padre guidata da Evangelista di Pian di Meleto, principale assistente del Santi e dunque strumento vivo di trasmissione del pensiero e della prassi artistica di Giovanni a Raffaello.

20 Su questo poema in terza rima, meglio noto come Cronica rimata, incompiuto (1478-1494): il fondamentale Michelini Tocci 1985; Varese 1988, 26-48; e più recenti contributi nei volumi su Giovanni Santi sopra citati.

${ }^{21} \mathrm{Nel}$ Libretto veneziano (Venezia, Gallerie dell'Accademia, Gabinetto dei disegni e delle stampe), «testimonianza indiretta del primissimo laboratorio mentale raffaellesco» (Farinella 2016), risalente agli inizi del Cinquecento, tra i disegni e gli schizzi di Raffaello, copiati, trasmessi e sistemati da un artista a lui prossimo, vi sono alcuni dei ritratti dello Studiolo, tra i quali il ritratto di Omero: https://arcade.nyarc.org/ record=b1109575 $\sim$ S7; immagine digitalizzata del foglio $(22,8 \times 16,4 \mathrm{~cm}$.): https://digitalcollections.frick. org/digico/\#/details/bibRecordNumber/b11095751/Photoarchive; Ferino Pagden 1982, 134-157.

22 Sul Tempietto, sull'ispirazione iconografica per le Muse (De gentilium deorum imaginibus di Lodovico Lazzarelli), sulle sue relazioni con la Cappella del Perdono e di entrambe con lo Studiolo, è fondamentale Wedepohl 2009. Sul Tempietto, inoltre: Clough, 1999, 63-70; Macioce, 1999, 71-76; Guidobaldi 2018, 479-498 e, sulle Muse nel Quattrocento, Ead. 1992, 15-24; Valazzi 2018, anche per il riallestimento temporaneo, in occasione della Mostra Giovanni Santi. "Da poi ... me dette alla mirabil arte de pictura», delle otto tavole conservate nel Tempietto (tavole a olio; $80,5 \times 37,5 \mathrm{~cm}$. Galleria Corsini, Firenze). La tavola con Talia e quella con Apollo (rispettivamente 80,5 × $51 \mathrm{~cm}$.; 81,5 × 37,3 cm. Galleria Corsini, Firenze) sono attribuite a Timoteo Viti che le eseguì verosimilmente tra il 1506-1508, al tempo di Guidubaldo da Montefeltro. 
‘percorso reale e simbolico〉 che dallo studiolo, attraverso la scala elicoidale, conduce nella zona sacra del Palazzo», rileva Nicoletta Guidobaldi. ${ }^{23}$ Almeno a sei delle nove Muse (1480-1490 circa) del ciclo pittorico del Tempietto aveva lavorato Giovanni Santi, che fu probabilmente tra gli ideatori del progetto iconografico, e che alle Muse aveva dedicato, negli stessi anni, versi nel citato poema La vita e le gesta di Federico. ${ }^{24}$

Questo luogo era uno spazio cultuale dedicato alla poesia e alla musica, incarnate con varietà di accenti e significati dalle Muse, tra rocce, fonti e ruscelli del Parnaso, e da Apollo, ${ }^{25}$ accompagnate molto probabilmente dalla Poesia stessa: pare che così debba intendersi la figura femminile seduta, dipinta in una delle tavole disperse del Tempietto. La convincente ipotesi si basa sul testo di riferimento del ciclo pittorico, il De Gentilium Deorum Imaginibus di Lodovico Lazzarelli (1470), e sulle correlate miniature, esemplate sui cosiddetti Tarocchi del Mantegna, ${ }^{26}$ tra le quali la Poesia è «ritratta come una fanciulla coronata d'alloro, seduta in un locus amoenus, che tiene un flauto diritto con la sinistra mentre con la destra versa da una brocca l'acqua della fonte Castalia che nutre l'ispirazione dei poeti». ${ }^{27}$ La sacralità di questo luogo era dichiarata nella frase che scorreva lungo le pareti del portico-vestibolo comune all'altro ieratico spazio, la Cappella del Perdono, ${ }^{28}$ e attraverso il vincolo architettonico e ideale fra i due inscindibili sacella, consacrati uno a Dio, uno alle Muse, veniva affermata e promossa «la concezione ficiniana nella quale filosofia platonica e religione cristiana non sono opposte, ma perfettamente integrate». ${ }^{29}$

L'Omero Smirneo dello Studiolo e le Muse del Tempietto urbinate non ebbero una diretta e visibile influenza nella elaborazione di Omero e del corteggio apollineo delle Muse nel Parnaso vaticano ma, mentre Raffaello lavorava a questo ambizioso affresco, dagli strati più remoti della memoria, l'uno e l'altra dovettero senza dubbio riaffiorare, non tanto come modelli figurativi quanto come riferimenti culturali e concettuali. Omero il primo poeta, il primo laureatus, il cieco veggente, la trasmissione dell' Iliade attraverso il libro (dipinto tra le sue mani e circolante nella corte del Duca di Montefeltro per mano di Bessarione), da un lato; dall'altro: Apollo e le Muse, immerse nello sfondo naturalistico del Parnaso condiviso con il dio, il platonismo ficiniano, che aleggiava nel Tempietto urbinate, l'Apollo musico con una «lira da braccio», «strumento quasi divino» che, al contempo, rendeva il dio simile a «un cantore improvvisatore», ${ }^{30}$ la Poesia laureata e seduta, latrice di ispirazione poetica.

${ }^{23}$ Guidobaldi 2018, 482.

24 «Nove vergin succinte iscalze e isnelle / Coi capei sparse d'or cum legiadria, / Le qual, sopra natura excelse e belle, / Coi segni in man venian cantando versi» (V, 92-95), in Guidobaldi 2018, 492.

25 Sulla consuetudine di decorare studioli e spazi privati con Apollo e le Muse, attestata a partire dalla metà del XV secolo in centri dell'Italia settentrionale, come Mantova, con il cosiddetto Parnaso di Andrea Mantegna (studiolo di Isabella d'Este; 1497 . Tempera su tela; 54, $6 \times 70,7 \mathrm{~cm}$. Paris, Musée du Louvre), rinvio a Cavallaro 2016, 479-480 e alla puntuale e aggiornata bibliografia (ibidem, 479, n. 13). Sulla centralità della musica negli studioli e in particolare nello Studiolo urbinate, nel Tempietto delle Muse, nell'ideologia culturale e politica di Federico da Montefeltro e, ancor prima, nella sua educazione, Bortoletti 2012, 60-63.

${ }^{26}$ Cavallaro 2016, 480, 482 (e n. 24 per la bibliografia), 484.

${ }^{27}$ Ipotesi e citazione: Guidobaldi 2018, 493, 497, cui si rinvia anche per la proposta di ricostruzione virtuale del Tempietto (ibid., 497, fig. 15), in cui la Poesia appare sulla parete di fondo, al centro, tra Atena e Apollo.

${ }^{28}$ BINA VIDES PARVO DISCRIMINE / IUNCTA SACELLA / ALTERA PARS MUSIS / ALTERA / SACRA DEO EST.

29 Guidobaldi 2018, 482.

${ }^{30}$ Guidobaldi 2018, 479-480, 482; per la citazione, 490. 
La presenza dell'universo artistico e intellettuale urbinate in Raffaello, mentre dipingeva il Parnaso e gli altri affreschi della Stanza della Segnatura, è provata dal ritratto del padre nella Scuola di Atene, accostato al proprio autoritratto. L'importanza del rapporto discepolo-maestro, affermata in molte delle relazioni in essere nella Scuola, trova espressione, intima e profonda, nella vicinanza dei volti dei due artisti urbinati. ${ }^{31}$ Con la parziale sovrapposizione dei visi e lo sguardo rivolto al padre, Raffaello dichiara la prima fonte del suo sapere pittorico, del suo estro poetico e soprattutto della sua dimensione intellettuale. Forse omaggia anche le capacità ritrattistiche del padre $;{ }^{32}$ certo valorizza una eredità, che avvicina idealmente non solo due artisti multiformi ma anche le due realtà artistiche e culturali dove padre e figlio avevano dimostrato e affermato a pieno la loro versatilità artistica e culturale: l'Urbino ducale e la Roma di Papa Giulio II.

Che questo legame fosse conosciuto, riconosciuto e, si potrebbe dire, approvato in Vaticano è chiaro dalle stesse parole del Pontefice che, nel Motu proprio del 4 ottobre 1511, con cui nomina Raffaello Brevium Scriptor, scrive così: Dilecto filio Raphaeli, Johannis de Urbino scolari, Urbinatensi pictori in palatio nostro. ${ }^{33}$

\section{Numine afflatur. La Poesia e il Parnaso nella Stanza della Segnatura.}

Originario di un borgo del Ducato di Urbino e parente di Raffaello, Donato Bramante fu figura chiave per l'accesso del giovane artista nella Roma di Giulio II (1503-1513), un Papa bifronte: da un lato, guida di un Papato imperiale pensato come erede ideale della Roma di Giulio Cesare, dall'altro, promotore di scoperte d'antichità, formidabile collezionista, perspicace e sensibile mecenate d'arte.

La particolare intesa tra Giulio II e Bramante, forte della caleidoscopica genialità dell'artista - architetto, pittore, cantore, amante della letteratura -, fu propizia all'arrivo di Raffaello a Roma nel 1508, ${ }^{34}$ con l'incarico di dipingere la Disputa del Sacramento (Trionfo dell'Eucarestia) nella Stanza della Segnatura (1508-1511), spazio di meditazione culturale e autocelebrazione. ${ }^{35}$ Pensata, con le Stanze dell'Incendio di Borgo, di Eliodoro e della Sala di Costantino, come ampliamento di rappresentanza dell'appartamento privato del Papa, era adibita a studiolo pontificio o biblioteca. ${ }^{36}$

31 A ridosso del ritratto della Scuola, Raffaello dipinse il volto del padre, una miniatura (1507) di cui si conserva una descrizione settecentesca di Giuseppe Bencivenni Pelli, direttore della Galleria degli Uffizi (Efemeridi, s. II, IX, maggio 1781), Farinella 2016.

32 Varese 2018, 79.

${ }^{33}$ http://irds-project.org/doc/1417/; Di Teodoro/Farinella 2017, 421; Farinella 2016.

${ }^{34}$ Bellosi/Rossi 1986, 582, 612, 925; Farinella 2016. Anche i Della Rovere, e in special modo Francesco Maria che aveva sostenuto Raffaello a Firenze, ebbero molto probabilmente un ruolo attivo nella venuta di Raffaello a Roma.

35 Nell'aprile del 1508, Raffaello tenta di avere l'incarico per dipingere una stanza ma si è incerti se questa fosse ancora quella dell'Udienza di Palazzo Vecchio o già quella della Segnatura, Frommel 2017, 13. Sicuramente il 13 gennaio 1509 Raffaello ricevette 100 ducati per gli affreschi della Stanza della Segnatura (per il testo del documento, con bibliografia: http://irds-project.org/doc/1952/). La bibliografia sulla Stanza è copiosa. Si vedano, in particolare e anche per altre indicazioni bibliografiche: Emiliani/Scolaro 2002; Joost-Gaugier 2002; Cannata Salamone 2002, 35-59; Taylor 2009, 103-141; Farinella 2014, 352-365; Frommel 2017, 14-39; Agosti 2017, 523-530; Casadei/Farinella 2017, 59-61.

${ }^{36}$ Autori e titoli dei libri del Pontefice, forse collocati in armadi posti al di sotto delle pareti affrescate, sono 
La Disputa del Sacramento (parete Ovest), le Virtù Cardinali e Teologali e la Legge (Sud), la Scuola di Atene (Est), il Parnaso (Nord) manifestano, mediante la trattazione, il dialogo, la meditazione e la scrittura, le attività della Teologia, della Giustizia, della Filosofia e della Poesia che, sub specie di figure allegoriche, dominano la Stanza dai tondi della volta quadripartita. ${ }^{37}$

Gli affreschi, pur non concepiti come narrazione continua, vanno compresi come un ciclo. Esistono, infatti, relazioni tra le quattro pareti e rapporti interni alle stesse, relazioni tra gli affreschi e le immagini sulle volte in linea con quella unità sempre ricercata da Raffaello, non solo nella Stanza ma in generale nei suoi lavori.

Chi accedeva alla Stanza della Segnatura andava con lo sguardo e con la mente dalla Teologia metafisica alla Poesia alla Filosofia alla Giustizia. Raffaello, Bramante e Dante, nelle vesti del Virgilio dantesco, guidano l'homo viator del Rinascimento alla contemplazione dei primi tre affreschi, dove appaiono più volte, e lo affidano, nell'affresco-epilogo della Giustizia, al solo Papa, lì dominante.

In particolare, Dante ${ }^{38}$ è trait d'union fra la Disputa (margine destro) e il Parnaso (margine sinistro); Raffaello fra la Scuola di Atene (margine destro) e il Parnaso (margine sinistro).

Oltre agli <uomini illustri〉 c’è un dio, pagano, a tessere una rete di relazioni: Apollo. Ricorrente anche nella volta, fra il tondo della Teologia e della Poesia, nel riquadro maggiore con la rappresentazione della mitica contesa musicale con Marsia, ${ }^{39}$ Apollo è figura-ponte tra il Parnaso e la Scuola di Atene, qui reso come immagine scultorea che inquadra, a sinistra, il simposio dei filosofi, nel Parnaso, quale asse della composizione. La stessa vocazione musicale del dio, presente già nel detto riquadro, unisce ulteriormente Scuola e Parnaso: nella Scuola, essa è data dalla scelta del tipo scultoreo antichizzante dell'Apollo citaredo, nel Parnaso dall'Apollo suonatore di lira da braccio.

La musica connota, in parte, anche l'allegoria della Poesia, in luminosa veste bianca e manto blu intenso. Da una vela della volta, in una posizione chiave al pari delle altre allegorie e rispetto al Parnaso, soprattutto ad Apollo, appare tenendo in mano una cetra, ${ }^{40}$

in parte noti da due inventari stilati da Romolo Mammacini, custode della libreria Vaticana, nel 1512. Gli inventari sono stati commentati e ripubblicati da Taylor 2009, 116-118; 135-141, anche per la precedente bibliografia. Sulla incertezza della funzione, biblioteca, appunto, o studiolo, tra gli ultimi: Casadei/Farinella 2017, 60; Agosti 2017, 523-530, anche per il puntuale status quaestionis.

37 La volta della Stanza, decorata con tondi allegorici e quadri narrativi, si data quasi certamente al 1511.

38 Dante era poeta necessario e ineludibile nel Parnaso per la statura poetica, l'opera, l'incidenza avuta da certi passi della Divina Commedia nell'elaborazione dell'affresco, la riabilitazione dei poeti, considerati invece mistificatori della verità da Padri della Chiesa e filosofi, come Girolamo, Tommaso e Platone, centrali chi nella Disputa, chi nella Scuola di Atene. Ecco che la funzione di collegamento tra la Disputa e il Parnaso data a Dante opera anche nella direzione di un 〈recupero〉 dei poeti e della poesia.

39 Il mito è nei versi iniziali del Paradiso di Dante (Par. I, 13-21: «O buono Appollo, a l'ultimo lavoro / fammi del tuo valor sì fatto vaso, / come dimandi a dar l'amato alloro. / Infino a qui l'un giogo di Parnaso / assai mi fu; ma or con amendue / m’è uopo intrar ne l'aringo rimaso. / Entra nel petto mio, e spira tue / sì come quando Marsïa traesti / de la vagina de le membra sue.»; Casali 2019, 25-47; miniatura della metà del XV secolo di Giovanni di Paolo, manoscritto della Divina Commedia, London, British Library, Yates Thompson 36, f. 129; http://www.bl.uk/manuscripts/Viewer.aspx?ref=yates_thompson_ms_36_fs001ar; Pope-Hennessy 1993) e, negli stessi anni della Stanza della Segnatura, nell'affresco di Baldassarre Peruzzi, Il supplizio di Marsia, nella parete Ovest della Sala del Fregio della Villa Farnesina a Roma, dove aveva lavorato anche Raffaello (Mercalli/Pagliai 1983, 32-38).

40 Se, come riteniamo, è corretta l'ipotesi avanzata da Nicoletta Guidobaldi sulla centralità della Poesia nella parete di fondo del Tempietto delle Muse a Urbino (supra, p. 212), questa potrebbe costituire un interes- 
cordofono che si ritrova nel sottostante affresco, retto da Tersicore in una variante organologica. Un libro, chiuso nell'altra mano, avvicina la Poesia a Clio.

Tra le quattro allegorie, la Poesia è la sola ad avere natura alata, segno di affinità con gli angeli, mentre condivide con i poeti la corona d'alloro. Ai lati, due tabulae ansate con le parole numine afflatur fissano l'imperituro soffio divino da essa ispirato e sintetizzano la vocazione divina del poeta. Si è pensato che questa icastica espressione fosse una rielaborazione di analoghe locuzioni impiegate da Cicerone, ${ }^{41}$ Virgilio $^{42}$ e Petrarca, suggerita a Raffaello da Tommaso Inghirami, suo amico, umanista tra i principali consiglieri e ispiratori - se non il principale - del programma iconografico delle Stanze e, dal 1510, Prefetto della Biblioteca Vaticana. ${ }^{43} \mathrm{Nel}$ discorso di ringraziamento, pronunciato in Campidoglio l'8 aprile 1341, per aver ricevuto la corona d'alloro, il Petrarca laureatus espresse l'origine divina dell'ispirazione poetica con le parole quasi divino quodam spiritu afflari. ${ }^{44}$ Pensiamo che questo discorso, noto come Collatio laureationis, ${ }^{45}$ sia fonte preziosa per ricostruire la genesi e la concezione del Parnaso vaticano. Non sembra, infatti, che Raffaello abbia tratto diretta ispirazione da un preciso modello iconografico, ${ }^{46}$ anche se più che una suggestione dovette giungere all'artista dal Parnaso del Tempietto urbinate - il suo primo Parnaso -, sviluppato per quadri distinti, appena uniti dalla comune ambientazione naturale del mitico monte e dalla condivisa presenza, in ogni tavola, di versi tratti da un canto sulle Muse ${ }^{47}$ e spunti dovette avere anche dal De Gentilium Deorum Imaginibus. ${ }^{48}$

sante precedente per l'enfasi della Poesia nella Stanza vaticana. Sulla rilevanza della musica nella 〈corte> di Giulio II, Moppi 2010, 295-322.

${ }^{41}$ Cic. Arch. 8,18: Atque sic a summis hominibus eruditisque accepimus, ceterarum rerum studia et doctrina et proeceptis et arte constare, poetam inflari. Il sottolineato è mio. La prospettiva ciceroniana sull'ispirazione divina dei poeti fu fatta propria da Inghirami nel suo commento In Poeticam Horatii (Vat. lat. 2742).

${ }^{42}$ Verg. Aen. 6,50-51: nec mortale sonans, adflata est numine quando / iam priore dei [...]. Il sottolineato è mio. Il poeta, con adflata est numine, descrive lo stato di trance della Sibilla.

${ }^{43}$ Su Inghirami, detto Fedra (o Fedro), per la sua magistrale interpretazione dell'omonima eroina nell' Hippolitus di Seneca: Guarino 2010, 353-355, 360; Bortoletti 2012, 57, n. 17; Farinella 2014, 358; Frommel 2017, 24. Altri nomi di ispiratori sono stati proposti: i più ricorrenti, Egidio da Viterbo, Giovanni Battista Casali, Pietro Bembo, Baldassarre Castiglione (si veda la bibliografia sulla Stanza della Segnatura, supra, p. 213, n. 35). A proposito del numine afflatur, Christoph Luitpold Frommel ha rilevato che afflatur, avendo molte lettere della firma dell'artista «Raffae. Ur», sarebbe anche «un gioco di lettere come quello che si trova nell'antependio della Disputa» (Frommel 2017, 34).

${ }^{44}$ Petr. Collat. laureat. 1,7,30.

${ }^{45}$ Wilkins 1943, 155-197; Feo 1990, 186-203; Maggi 2004, di cui si segue il testo; Cannata/Signorini 2014, 459-464.

46 Sulla consuetudine di decorare studioli e spazi privati con Apollo e le Muse, attestata a partire dalla metà del XV secolo in centri dell'Italia settentrionale, come Mantova, con il cosiddetto Parnaso di Andrea Mantegna (studiolo di Isabella d'Este; 1497 . Tempera su tela; 54, $6 \times 70,7 \mathrm{~cm}$. Paris, Musée du Louvre), rinvio a Bortoletti 2012, 68-69; Cavallaro 2016, 479-480 e alla puntuale e aggiornata bibliografia (ibidem, 479, n. 13).

${ }^{47}$ Il carme era ritenuto - erroneamente - di Virgilio ( $P$. V. Maronis de musarum inventis) o di Ausonio, con qualche incertezza. Guidobaldi 2018, 485-486, anche per la bibliografia. La disposizione urbinate delle Muse e i loro attributi, elementi iconografici delle Muse dei citati Tarocchi del Mantegna vennero ripresi, con varianti, nel ciclo delle Muse della Magliana, all'interno della Villa di Leone X, realizzato da Gerino da Pistoia in anni successivi al 1511. Cavallaro 2016, 483-487.

48 Il testo di Lazzarelli aveva ispirato le citate Muse del Tempietto del Palazzo Ducale ma era verosimilmente noto e circolante a Roma tra la fine del XV e l'inizio del XVI secolo, Cavallaro 2016, 484, n. 28. 
Questo verosimile background del Parnaso dovette ricevere linfa proprio dalla Collatio, testo noto nella Roma giuliana ${ }^{49}$ e implicitamente integrato nel Parnaso della Stanza mentre più esplicitamente le incoronazioni poetiche vennero integrate nel cortile vaticano del Belvedere, il cui «spazio del nuovo giardino delle statue $[\ldots]$ usurpava l'incoronazione poetica, prerogativa del colle capitolino». ${ }^{50}$ Sono vari i temi, i segni, i personaggi che attraversano la Collatio laureationis, sorta di inno alla Poesia, ${ }^{51}$ e che si ritrovano nell'affresco della Stanza e sono varie pure le affinità di senso. Su tutte, vanno rilevate: la celebrazione della poesia nell'accezione sopra ricordata; le valenze metaforiche dell'alloro per la poesia, la funzione di tutela dei libri dai danni del tempo esercitata da questa pianta - funzione quanto mai consona a una biblioteca, quale era probabilmente la Stanza -; la figura di Omero, divinarum omnium inventionum fons et origo, il quale, sub poetici nube figmenti, verum sapientibus intelligi dedit e poetarum princeps. ${ }^{52}$

Nella parete del Parnaso (cronologia oscillante tra 1509-1510 e 1510-1511 - ipotesi, quest'ultima, più fondata), ${ }^{53}$ sovrastata dall'allegoria della Poesia, il fuoco della composizione è costituito dall'archetipo del laureatus, il divino Apollo. Il serto d'alloro è anche sulla testa dei poeti, a cominciare da quella di Omero: grazie alla corona, diventano tutti partecipi di quella divinazione di cui Apollo laureato è «dio». ${ }^{54} \mathrm{Nel}$ Parnaso, le <poetiche» piante di alloro sono disposte in modo tale da evocare un bosco e, di nuovo, pare esserci un riferimento alla Collatio, esattamente all'ampia parte che Petrarca dedica all'alloro della sua corona, ${ }^{55}$ il cui incipit è la citazione del lauri nemus dei Campi Elisi dall'Eneide. ${ }^{56} \mathrm{La}$ presenza di Virgilio e del suo poema ricorre più volte: nella menzionata epigrafe numine afflatur, nel ritratto ideale del poeta - alle spalle di Omero -, nel monocromo con Augusto che salva l'Eneide dalle fiamme, dipinto al di sotto del Parnaso, e all'ingresso del Cortile del Belvedere, nelle parole procul este profani. ${ }^{57}$

Il dio Apollo, che siede al centro dell'affresco come Cristo nella Disputa del Sacramento, ha il volto rivolto verso l'alto in atteggiamento estatico e accompagna il canto poetico con una viola rinascimentale a nove corde, un tipo di strumento ritenuto adatto «al coro delle Muse», ${ }^{58}$ secondo quanto scriveva, nel XVI secolo, il compositore, liutaio, teorico della musica Vincenzo Galilei. La nuova attribuzione della «lira o viola da braccio» ad Apollo come mostra, fra gli altri, il Musagete del Tempietto urbinate ${ }^{59}$ - rifletteva però soprattutto

49 Ad esempio, Guarino 2010, 351.

${ }^{50}$ Guarino 2010, 357.

${ }^{51}$ Sulle valenze della Collatio, sui fondamentali nessi con Parnaso e Muse in questo e in altri testi del Petrarca: Cannata/Signorini 2014, 459-464; Geri 2014, 617-631.

52 Petr. Collat. laureat. 9,5; 17,52-53; Battaglia Ricci 2018, 18, 33, 39 per il riferimento alla cosiddetta lettera a Omero di Petrarca (Foti 2007). Lo stesso Petrarca è tra i poeti laureati nel Parnaso vaticano, Kirkham 2015, 462-463; Casadei/Farinella 2017, 64. Sui valori dell'alloro in riferimento alla poesia, ai poeti, ad Apollo, Clements 1955, 789-790, 793.

53 Tra i molti: Watson 1987, 127-148; Id. 1988, 113-141; Cannata Salamone 2002, 35-59; Unglaub 2007, 14-17; Frommel 2017, 32-35; Casadei/Farinella 2017, 61.

${ }^{54}$ Petr. Collat. laureat. 11,14,62-63.

55 Petrarca è tra i poeti dipinti da Raffaello nel Parnaso e nei sonetti composti dal pittore (infra, p. 217) vi sono stilemi petrarcheschi. Scrivano 1983, 272.

${ }^{56}$ Verg. Aen. 6,658.

57 Verg. Aen. 6,258. Farinella 2014, 360.

58 «È applicato l'Ennecordo al coro delle Muse», Galilei 1581, 116.

59 Guidobaldi 2018, 490. Stesso strumento è suonato dall'Apollo della citata villa della Magliana (supra, p. 215, n. 47), che ha per modello l'Apollo del Parnaso, Cavallaro 2016, 480-481, 486-487, 492, figg. 3-4. 
la circolazione di questo strumento «nel primo Cinquecento»: essa «era legata alla moda dei poeti di recitare i propri versi accompagnandosi con la musica», ritenuta perfetta per «la recitazione e il canto di versi poetici», ovvero per «il 〈recitar cantando»». ${ }^{60}$

Il dio è circondato dalle nove Muse: quattro a sinistra, cinque a destra ${ }^{61} \mathrm{Il}$ primo consesso (Calliope, Erato, Polimnia, Euterpe) è contiguo ai sommi poeti, Omero, Virgilio, Dante. Fra tutti, Omero è il più vicino a Calliope, ${ }^{62}$ la Musa dell'epica che non può che avere la massima prossimità con il primo poeta epico e l'antonomastico. Omero alza lo sguardo al cielo, come Apollo, e contemporaneamente rivolge la parola poetica a un giovane alla sua destra, seduto, con un calamo in mano e fogli sulle ginocchia, in atto di scrivere quello che il poeta declama. Alle spalle di Omero, dietro a Virgilio, appare un personaggio che, per i tratti somatici, viene identificato con Raffaello. ${ }^{63}$ Questa figura di giovane cerca lo sguardo dell'osservatore del Parnaso, allo stesso modo del Raffaello del sicuro autoritratto nella Scuola di Atene. Si pone discretamente tra Virgilio, il vate della nascita di Roma, di quella città alle cui antichità Raffaello era legato, in modo viscerale e per più ragioni, ${ }^{64} \mathrm{e}$ Calliope, Erato, Polimnia. I volti di queste Muse sembrano rispecchiare il viso della donna amata dal pittore, ritratta ne La donna velata e a sua volta musa di sonetti d'amore, alla maniera del Petrarca, che Raffaello appuntò su bozzetti della Disputa e del Parnaso, segno di una propensione poetica - nota anche da altre testimonianze - e che verosimilmente indusse l'artista a dipingersi laureatus fra laureati. ${ }^{65}$

${ }^{60}$ Cavallaro 2016, 478, 480-481. Guccini 2012, 465-468, 471-473. Il paragrafo Raphael's Parnassus and the Apotheosis of cantare ad lyram, Wilson 2020, 398-405. Si è ipotizzato, inoltre, che l'immagine dell'Apollo del Parnaso sia stata ispirata da un preciso e amatissimo musico e cantore, in attività presso la corte di Giulio II, Giacomo Sansecondo. Bortoletti 2012, 52, 66.

61 Winternitz 1952-54, 359-388; Guccini 2012, 473-474.

${ }^{62}$ Nell'incisione del bolognese Marcantonio Raimondi (collaboratore di Raffaello a Roma dal 1510, Talvacchia 2013, 305-310), Parnaso-Raphael pinxit in Vaticano (1517-1520), relativa a una elaborazione iniziale dell'affresco (Casadei/Farinella 2017, 61; Davoli/Panizzi 2020, 17), Calliope è rivolta verso Omero, fissando con il poeta un rapporto più stretto che nell'affresco. Altri (ad esempio, Guccini 2012, 466) identificano Calliope con la Musa, seduta a sinistra di Apollo, con in mano un aerofono, per la quale va piuttosto accolta l'identificazione con Euterpe, Morelli 2007, 188-189.

${ }^{63}$ Così, fra gli altri, Giovanni Pietro Bellori 1825. Altri (come Unglaub 2007, 15; Casadei/Farinella 2017, 62) identificano il giovane laureato con Stazio, per il legame con Virgilio e l'incontro con Virgilio e Dante nel Purgatorio. La figura è assente nella incisione di Raimondi, appena ricordata.

${ }^{64}$ Il legame con le antichità, chiaro anche in vari punti degli affreschi della Stanza della Segnatura, ancor più in quelle di Eliodoro e dell'Incendio di Borgo - affresco della Battaglia di Ostia - (Farinella 2016), venne ufficializzato il 27 agosto 1515, quando Leone X nominò Raffaello Praefectus marmorum et lapidum omnium, Commissario delle antichità, dei reperti archeologici marmorei e delle epigrafi. Testo del documento di nomina papale: http://irds-project.org/doc/129/; Scrivano 1983, 273; Patetta 2014, 34-35. Tra le risonanze di questa nomina e dell'encomiabile svolgimento dell'incarico da parte di Raffaello, sempre più «artista-antiquario» (Farinella 2016) e sarcheologo», si leggano il componimento di Girolamo Leandro (1516; http://irds-project.org/doc/1437/); l'epigramma di Celio Calcagnini (1519; http://irds-project.org/ doc/2555/); un passo del Libro III delle Prose della volgar lingua di Pietro Bembo (1525; http://irds-project. $\mathrm{org} / \mathrm{doc} / 1947 /)$. Tra gli atti più significativi, anche per il valore pionieristico, è la celebre lettera di Raffaello, già «assai studioso di queste antichità», a Leone X (1519), scritta con Baldassarre Castiglione e il contributo di Angelo Colocci (http://irds-project.org/doc/2559/; http://irds-project.org/doc/2560/; http://irdsproject.org/doc/2561/). Sulla lettera a Leone X, da ultimo Terraroli 2020. Su Raffaello e l'antico, a partire dal 1515: Farinella 2016.

${ }^{65}$ Sui sonetti, sulla solida cultura letteraria di Raffaello, sulla costante frequentazione della intellighenzia romana, sulla relazione amicale e soprattutto culturale con Inghirami: Scrivano 1983, 265-285; Turner 2013, 6-11; Frommel 2017, 33. In particolare, il legame di Raffaello con la poesia è esplicitato visivamente nel 


\section{Stratigrafia di una immagine: il gruppo scultoreo del Laocoonte e l'Omero di Raffaello con il giovane rapsodo.}

La finestra attorno alla quale fu dipinto il Parnaso, dominato da Apollo, si apriva sul colle Vaticano, in antico legato al culto del dio, sul Cortile del Belvedere e sull'odeon. Anche il cortile bramantesco era segnato da Apollo, almeno dal 1508, quando accolse la famosa statua omonima - detta per questo, e tuttora, Apollo del Belvedere -, una delle più sensazionali scoperte d'antichità del Rinascimento e tra le punte di diamante della collezione del Cardinale Giuliano della Rovere, come noto, divenuto Papa Giulio II. ${ }^{66}$ Nel Cortile furono disposte anche altre sculture e pezzi d'eccellenza, messi in luce a Roma tra il XV e l'inizio del XVI secolo, come il celeberrimo gruppo statuario del Laocoonte: ${ }^{67}$ grazie ad esse il Cortile assunse l'aspetto e la funzione di uno spazio-museo e di uno spazio-teatro. ${ }^{68}$ In esso si svolgevano anche rappresentazioni teatrali, declamazioni e incoronazioni poetiche, tra cui la leggendaria e spettacolare del 1512, che ebbe tra i protagonisti l'allora prefetto della Biblioteca Vaticana (1510) e uno dei principali ideologi delle Stanze, il citato Tommaso "Fedra» Inghirami, che consegnò al Pontefice le corone d'alloro per i poeti. ${ }^{69}$ Questa celebrazione, che comprese anche recitazioni di versi da parte di fanciulli, vestiti da Muse, di allievi dell'accademia romana, come Vincenzo Pimpinella, in abito di Orfeo/ Apollo, e di figure come Raffaele Brandolini, noto anche per le doti da improvvisatore e la cecità, per le quali era considerato alter Homerus, ${ }^{70}$ fu il punto di convergenza del «ritorno del Parnaso», dell' «insediamento dei rituali accademici», della «trasfigurazione archeologica degli spazi vaticani». ${ }^{71}$

Il Parnaso della Stanza della Segnatura non era isolato, né in senso topografico nè semantico: nell'affresco - metaforicamente - e tra l'affresco e il Cortile del Belvedere prendeva vita il nuovo Parnassus di Giulio II e della sua corte rinascimentale nel segno di una ideale

ritratto dell'artista come poeta fra Dante, Petrarca, Poliziano, nel soffitto di una stanza (forse uno studio) accanto alla loggia di Villa Lante al Gianicolo, affrescata da Giulio Romano, allievo prediletto di Raffaello, per Baldassarre Turini, mecenate, umanista, appassionato d'antichità e amico dell'Urbinate (Merisalo 2016, 243-245). L'amore di Raffaello per la poesia e la vicinanza iconografica a Dante e Petrarca in veste di poeta accostano di nuovo Raffaello al padre: oltre ad aver composto la Cronaca rimata, Giovanni Santi recitava in piazza versi degli amatissimi Dante e Petrarca che, memorizzati grazie a una prodigiosa capacità mnemonica, gli fecero acquistare gran fama di cantastorie (Michelini Tocci, XI-XII, XXII).

${ }^{66}$ Sulla scoperta dell'Apollo del Belvedere e sulla storia ‘giuliana) della statua, Guarino 2010, 346, nn. 3-4.

${ }^{67}$ Della vasta bibliografia, tra gli studi fondamentali: Settis 1999. Più recente, Bejor 2007.

${ }^{68}$ In particolare: Winner/Andreae/Pietrangeli 1998; l'ottimo articolo di Guarino 2010, 345-364. Sono emblematiche, a proposito dello sspazio-museo', le parole dell'antiquario Andrea Fulvio nel descrivere il Mons Vaticanus e le sue meraviglie e nell'allusione all'amore di Giulio II per il bello. Scrive Fulvio: «Fece ancora una bellissima fontana con un giardino di aranci, circondato da un muro, al centro del quale c'è la statua di Nilo con i suoi attributi, e del Tevere con i due fanciulli che costruirono Roma che giocano con le mammelle della lupa. Da un lato ci sono poi la statua di Apollo con l'arco e le frecce e quella di Laocoonte virgiliano con i due figli annodati dalle spire dei serpenti, tratta da un solo marmo. C’è poi la statua di Venere che guarda accanto a sé il piccolo Cupido e Cleopatra posta accanto alla fonte, simile ad una donna che sta per perdere i sensi. E infatti il complesso animo di Giulio (arduus Iulii animus) ardeva per tutte le cose eccellenti (ad omnia praeclara ardebat)». La citazione dal II libro delle Antiquitates Urbis per Andream Fulvium antiquarium, nuperrime editae - De monte Vaticano et eius ornamentis, è tratta da Maffei 1999, 158-159.

${ }^{69}$ Benedetti 2004; Guarino 2010, 350-355; Bortoletti 2012, 56-59.

70 Bortoletti 2012, 57-58; Guccini 2012, 477.

71 Guarino 2010, 350. 
continuità fra la cultura antica e quella dell'età dell'alter Iulius, infaticabile tessitore di fili tra le «proprie imprese culturali, politiche e militari [...] e quelle compiute da Giulio Cesare», ricondotte e giustificate "entro un'ottica cristiana». ${ }^{72}$

Il Parnassus giuliano era dichiaratamente memore della centralità di Apollo e della prima voce poetica, quella di Omero, il primo poeta ispirato dal dio e il poetarum princeps: così appare dalla postura del volto, dalla collocazione nello spazio e dal rapporto con gli altri poeti.

A differenza dei poeti contemporanei a Raffaello raffigurati nel Parnaso, come Jacopo Sannazaro, ${ }^{73}$ il volto di Omero non poteva che essere affidato a un ritratto di ricostruzione, ${ }^{74}$ a quella tipologia di ritratto che induce l'artista, preso dal desiderio di entrare in relazione con gli «Uomini illustri» del passato, a immaginare visi di cui sono ignote le reali sembianze. Il ritratto di ricostruzione è una immagine fittizia ma restituisce - o almeno questo è il suo intento - l'idea maturata, nel tempo, spesso attraverso la lettura dei testi o la tradizione orale, attorno a uno spirito magno.

La prassi del ritratto di ricostruzione riguarda Omero da sempre e di essa parla in modo esplicito Plinio, la cui Naturalis Historia era ben nota nei primi anni del Cinquecento. L'autore latino, indicando anche uno tra i principali luoghi di esposizione di questo genere di ritratto, scrive:

non va dimenticata neppure la recente trovata di dedicare nelle biblioteche ritratti [...] a coloro le cui anime immortali parlano in quei medesimi luoghi; anzi, si raffigurano anche ritratti immaginari, e i desideri creano volti non tramandati, come è accaduto nel caso di Omero (etiam quae sunt finguntur, pariuntque desideria non traditos vultus, sicut in Homero evenit). ${ }^{75}$

Sempre nella Naturalis Historia, Plinio menziona un'importante opera di Varrone sull'arte del ritratto, Imagines o Hebdomades (39 a.C.), dove viri illustres, disegnati, dipinti, accompagnati da un elogio, erano disposti in gruppi. ${ }^{76}$ Questa dislocazione fu adottata da Raffaello nel Parnaso quando - ripetiamo - l'opera di Plinio era conosciuta e diffusa. ${ }^{77}$

Anche nel trattato rinascimentale De Sculptura (1504) di Pomponio Gaurico è espresso un concetto analogo a quello pliniano e proprio in relazione a Omero: "Gli scultori la [scil.: la fisiognomica] terranno in grande considerazione, in quanto grazie ad essa potranno rappresentare con grande facilità quello stesso Omero, che tanto desideriamo vedere, e gli stessi sapienti della Grecia». ${ }^{78}$ Con riferimento a questo passo, Lina Bolzoni ${ }^{79}$ rileva come

72 Farinella 2014, 349.

73 Casadei/Farinella 2017, 62, 64-66, 68-69.

74 La Rocca 2011, 21-29; Parisi Presicce 2011, 121-136.

75 Plin. nat. 35,9. La traduzione è mia. Già in età ellenistica, oltre alle biblioteche, monumenti encomiastici, santuari, sedi di culto e studio, fra cui il famoso Homereion alessandrino, ospitarono ritratti di ricostruzione.

${ }^{76}$ Plin. nat. 35,11.

77 La conoscenza e circolazione di Plinio nat. 35 è dimostrata, ad esempio, dagli espliciti riferimenti nel De inventoribus rerum libri octo di Polidoro Virgili (1521). Passo e indicazioni bibliografiche: http://irdsproject.org/doc/2548/. Su Raffaello lettore di Plinio, Rinaldi/Falcucci 2010, 387-402. La conoscenza, seppure indiretta, delle opere di Attico e di Varrone ebbe una influenza notevole sugli studi Cinquecenteschi di iconografia: Inlustrium virorum ut exstant in urbe expressi vultus di Achille Stazio (Roma, 1569); Imagines et elogia virorum illustrium di Fulvio Ursino (1570). Varese 2018, 79.

78 Il passo del De Sculptura è citato da Bolzoni 2012, 25, con testo latino - di cui segnalo il verbo desideratur, così vicino al termine desideria impiegato da Plinio -, con traduzione e rinvio all'edizione di André Chastel e Robert Klein.

79 Bolzoni 2012, 24-25. 
sia significativo che "Omero venga citato per primo, come primo oggetto" del «desiderio che consiste nel poter disporre anche del ritratto, dell'immagine fisica dei grandi del passato». E aggiunge: «La fisiognomica si presenta [...], all'inizio del Cinquecento, come una specie di scorciatoia per realizzare quel desiderio di cui parlava Plinio, come la tecnica cui si chiedono gli strumenti per tradurre in un ritratto visibile quel ritratto invisibile che $\mathrm{i}$ testi trasmettono» e che trova negli studioli i luoghi ideali. "Se leggere vuol dire incontrarsi con l'autore, vuol dire evocarne i lineamenti e la presenza con una intensità quasi fisica, allora la presenza del suo ritratto aiuterà il compimento del rito, e parole e immagini si appoggeranno le une alle altre».

Raffaello, per il suo ritratto omerico, non potè considerare i ritratti di ricostruzione greci e romani, ${ }^{80}$ dal momento che quasi sicuramente non li conosceva. Neppure i precedenti più prossimi, tuttavia, si intravedono per allusione iconografica nell'Omero della Stanza: nulla vi è dell'immagine d'Omero dello Studiolo urbinate. Senza dubbio, però, è anche attraverso quella prima memoria artistica e culturale sul poeta greco che Raffaello elabora il 〈suo〉 Omero: l'idea della cecità, della natura di divino poeta, di veggente, dell'età avanzata, del libro (ossia, del testo scritto), approda nell'Omero di Raffaello attraverso una dinamica stratificazione che, per l'artista urbinate, aveva il suo atto di nascita nell'Omero dello Studiolo della sua città natale. A questo primo strato se ne aggiunsero altri: modelli iconografici, riferimenti e stimoli letterari, di cultura orale, riletti dall' «onnivora curiosità intellettuale» ${ }^{81}$ di Raffaello, selezionati e assimilati con intelligente sensibilità, ripensati e rielaborati attraverso il filtro della propria concezione del ritratto ${ }^{82}$ e tenendo conto di un preciso contesto artistico, culturale e ideologico, il Parnassus pontificio, in cui Omero andava inserito come archetipo dei poeti.

Il volto del proto-poeta è rovesciato all'indietro; è colto in una ispirazione concentrata e tesa, contrapposta a quella estatica di Apollo; è fermato in un silente dialogo con il numen dal quale nasce la parola poetica, da accogliere e trasmettere. Raffaello fa proprio il tradizionale tratto omerico della cecità in una concezione e rappresentazione di Omero del tutto originale. Essa attinge moduli espressivi della statuaria patetica ellenistica, che l'Urbinate potè cogliere da una eclatante scoperta della Roma pontificia (1506), esposta con enfasi nel Cortile del Belvedere: il Laocoonte. ${ }^{83}$ Raffaello ebbe modo di osservare il gruppo statuario con molta accuratezza perché, nel 1507-1508, fu incaricato da Bramante di valutare la migliore copia, completa delle parti mancanti, eseguita da alcuni dei più talentuosi scultori dell'epoca. ${ }^{84}$ Poco prima di iniziare il Parnaso, Raffaello, non solo vide il Laocoonte ma, per onorare l'incarico ricevuto, lo studiò accuratamente, da vicino. ${ }^{85}$ Del volto del sacer-

${ }^{80}$ Eccellente panoramica sui ritratti di Omero dal V sec. a. C. al IV sec. d.C. è tracciata da Zanker 2009, 17-24, 182-190, 190-195, 217-221, 356, 358-361. Pregnanti considerazioni su alcuni di questi ritratti e su altri volti di Omero in Most 2004, 1-14. Indicazioni bibliografiche sui ritratti di Omero nell'antichità e nel Medioevo, Vendruscolo 2015, 154, n. 5; Pontani 2005, 1-26, per l'analisi di un ritratto bizantino di Omero, svolta tenendo conto dei principali precedenti iconografici.

${ }^{81}$ Farinella 2016.

82 Raffaello considerava il ritratto strumento "per conservare la memoria, simbolo dell'individuo»; immagine parlante - imago loquitur - con l'osservatore, che deve poter «decifrare stati d'animo, destino, intenzione» di chi è ritratto: Strinati 2001, 13-27.

${ }^{83}$ Maffei 1999, 99-113, 151-201.

${ }^{84}$ Tra gli artisti selezionati da Bramante, l'artefice della migliore ricostruzione fu Jacopo Tatti detto il Sansovino.

${ }^{85}$ In generale sullo studio dei modelli da parte di Raffaello, De Vecchi 2001, 29-38. 
dote troiano, riprese per Omero la concentrazione, ma opportunamente spogliata di quel sentimento di strazio e dolore che attraversava il pensiero di Laocoonte; risemantizzò la bocca aperta, non più per un urlo trattenuto, come nel troiano, ma per l'emissione di voce poetica. Raffaello mitigò molto l'accentuata inclinazione del viso di Laocoonte verso sinistra, troppo patetica per il ritratto del vate. ${ }^{86}$ Mantenne, invece, la barba dai fitti ricci, persino nel lieve biforcarsi sotto il mento, i baffi e la capigliatura, in Omero meno folta per via dell'età avanzata e soprattutto per dare risalto al serto di alloro. ${ }^{87}$

Anche per il corpo, la ricostruzione di Raffaello si differenzia molto dalle possibili ricostruzioni precedenti del corpo del poeta, ipotizzato spesso in piedi, ma vacillante a causa della cecità, oppure seduto, come nel citato rilievo ellenistico di Archelaos di Priene e nello Studiolo di Urbino. L'Omero della Stanza della Segnatura è stante, maestoso, saldo. La postura del piede destro, avanzato verso il giovane, e soprattutto il braccio proteso con fermezza verso di lui, che lo fissa e ascolta con attenzione mentre si accinge a scrivere, introduce una pregnante apertura nello schema del poeta. Il corpo è avvolto in un abito anticheggiante, composto da chiton e himation, giallo e blu. Attraverso il giallo Raffaello avvicina Omero alla Musa dell'astrologia Urania che, di spalle, chiude il gruppo delle Muse $;^{88}$ attraverso il blu, seppure di diversa nuance, accosta Omero alla Poesia, facendo di lui il solo, tra i poeti del Parnaso, a godere di questa consonanza cromatica e riconoscendogli unicità poetica anche per mezzo di questa soluzione iconografica.

Il corpo dell'Omero di Raffaello è sparlante> al pari del volto. Lo schema è un perfetto equilibrio di stabilità, ottenuta nella ferma impostazione della imponente parte sinistra del corpo e nella mano chiusa in un pugno, e di aperture, date dalla postura del capo proteso verso i cieli del Parnaso e dal braccio destro, aperto e disteso, come la mano, in direzione del giovane che sta per scrivere. Con questo schema Raffaello crea un Omero fermato nella consapevolezza della propria maestosità e maestà poetica, sovrastante tutti i laureati e, allo stesso tempo, un Omero teso ad un doppio dialogo: uno, con il numen ispiratore, che lo rende simile all'ispirato Apollo; l'altro con chi dovrà dare memoria al suo canto attraverso la parola scritta. Il ruolo di chi deve tramandare per iscritto la poesia è fondamentale, come mostra anche, nella volta della Stanza, l'allegoria della Poesia, che tiene un libro in mano ed è affiancata dalla parola scritta nella doppia epigrafe. La parola poetica ha dunque bisogno della scrittura e dei libri per poter essere trasmessa nel tempo.

Il fanciullo alla destra di Omero, seduto sulla roccia del Parnaso, si accinge a scrivere le parole ispirate del poeta. ${ }^{89}$ Sull'identità del giovane, per lo più considerato erroneamente un poeta, sono state avanzate varie ipotesi ${ }^{90}$ ma l'assenza di tratti distintivi poetici, fin

${ }^{86}$ Molto più somigliante al volto di Omero è il volto di Odisseo - che pure ha palesi affinità con quello di Laocoonte - del colossale gruppo scultoreo di Ulisse che acceca Polifemo (noto come Gruppo di Polifemo), opera degli stessi artifices del Laocoonte, Atenodoro, Agesandro, Polidoro (si veda più oltre in questo paragrafo). Raffaello non poteva però conoscere l'opera (ora nel Museo Archeologico Nazionale di Sperlonga), rinvenuta nel 1957 a Sperlonga, in una grotta nella villa marittima di Tiberio. De’ Spagnolis 2017.

87 Tra le iconografie poetiche e musicali della Stanza della Segnatura, anche nel citato quadro della volta con il mito di Apollo e Marsia (supra, p. 214), questi è dipinto da Raffaello secondo gli stilemi del Marsia noto dalla statuaria ellenistica, in particolare di scuola pergamena, mediata da copie romane.

${ }^{88}$ Guccini 2012, 474-475.

89 Sul giovane, Fornaro 2018, 1025-1042.

90 Tra queste spicca quella del poeta Ennio, ad esempio: Cannata Salomone 2002, 41; Casadei/Farinella 2017, 62. Approfondita discussione, nel paragrafo di Sotera Fornaro, Il giovane che scrive, in Fornaro/Viccei 2020, 32-33. 
dalla corona laureata, ${ }^{91}$ l'abito, la postura e analogie interne ad altri affreschi della Stanza della Segnatura, dove appaiono giovani scribentes o scripturi in relazione a personaggi più anziani che dettano, inducono a proporre una diversa lettura del giovane. Solo sul suo capo manca il serto di alloro e questa assenza non è irrilevante nel Parnaso vaticano, dove è stato ampiamente dimostrato che in hoc signo va posto il mestiere di poeta. In un simile contesto, un poeta senza corona laureata sarebbe una deroga incomprensibile. Anche nell'abito, il giovane fa eccezione: lui solo indossa una veste corta. Il colore in parte giallo è anche dell'abito di Omero e, con ovvie differenze, sembra che Raffaello abbia voluto sottolineare pure con questa similarità cromatica l'espressa relazione di funzioni e schemata fra il Poeta e il giovane. L'abito dello scrivano ha, poi, un interessante dettaglio di realismo, che contribuisce a restituire con più efficacia la funzione del fanciullo: la manica destra è tirata sopra al gomito, segno di cura a non volerla sporcare con l'inchiostro. Si è detto, e in parte dimostrato, che tra i dipinti della Stanza vi sono echi interni e alcuni riguardano proprio il <giovane di Omero che, per la funzione, mostra analogie con il giovane scrivente della Disputa del Sacramento e ancor più con quello che scrive sotto lo sguardo del filosofo ascetico accanto a Democrito nella Scuola di Atene..$^{92}$ A questo il «giovane di Omero può essere accostato anche per la postura - entrambi sono seduti con le gambe accavallate - e per l'abito corto. Ancora nella Scuola vi sono altre similarità, con il giovane discepolo di Euclide che, per fisionomia - specie volto e capigliatura - e per il dettaglio della manica accorciata della veste, rimanda al sgiovane di Omero».

Torniamo ora al volto del Poeta. L'affinità di questo con il volto di Laocoonte è stata già variamente colta, ${ }^{93}$ tuttavia restano aperte questioni essenziali: perché Raffaello sceglie il volto del Laocoonte per Omero? Quale idea di Omero vuole comunicare ai frequentatori della Stanza della Segnatura, che visitavano, o potevano visitare, anche il Cortile del Belvedere, dove era esposto il gruppo scultoreo? Nasce da Raffaello l'idea di ispirarsi al Laocoonte o è suscitata, se non richiesta espressamente, da Giulio II? E in questo caso, dal solo Papa o da uno dei consiglieri della creazione della Stanza, penso soprattutto a Inghirami, che tanta parte ebbe nell'ideazione iconografica e nel lessico della Stanza? Quanta autonomia ha avuto Raffaello nel dipingere Omero, la stessa, ampia, lasciatagli per la Scuola di Atene? In questa sede affronto solo alcuni di questi temi e senza pretendere di esaurirne la problematicità. ${ }^{94}$

Punto di partenza è cogliere il significato che Laocoonte aveva per il Papa e il suo entourage perché è nella Roma di Giulio II, prima che nella duttile intelligenza visiva di Raffaello, che si deve collocare la scultura, tuttavia tenendo come importante ipotesto il dialogo sempre vivace tra il Papa-committente e i suoi artisti. Per affrontare questo tema essenziale

${ }^{91}$ Già Gutman 1958, 33: «Rechts und links von der Zentralgruppe erblicken wir Gruppen von Gestalten, die durch ihre Lorbeerkränze als Dichter gekennzeichnet sind. Nur ein Nichtdichter ist unter ihnen, der Schreiber, der Homer's Gesänge aufzeichnet und der [...] seine Gegenstücke in der Disputa und der Schule von Athen findet».

${ }^{2}$ Nella Scuola di Atene e nella Disputa, Raffaello propone raffigurazioni di vari atti della scrittura: copiare testi, scrivere sotto dettatura, semplicemente scrivere.

93 Venturi 1889, 101; Emiliani/Scolaro 2002, 178-183; Cannata Salamone 2002, 40; Moormann 2004, 137; Rijser 2012, 149-150.

${ }^{94} \mathrm{~A}$ un approfondimento di tali questioni, a risposte agli interrogativi qui avanzati preliminarmente ma inevasi, ad altre questioni ora solo embrionali, dedicheremo ampio spazio nella monografia in preparazione (supra, p. 209, n. 9). 
sono illuminanti e del tutto condivisibili le considerazioni di Sonia Maffei e di Vincenzo Farinella. In La fama di Laocoonte nei testi del Cinquecento, Maffei ${ }^{95}$ evidenzia che la morte del sacerdote troiano è

simbolo della caduta di Troia ma anche sacrificio che permette la fuga di Enea e la fondazione di Roma. Il mito troiano prefigura così nel dolore di Laocoonte lo splendore della Roma augustea. Non meno del giudizio di eccellenza di Plinio il Vecchio ${ }^{96}$ l'altezza della poesia virgiliana contribuisce [...] a collocare il Laocoonte di marmo scoperto nel 1506 all'apice del giudizio dei contemporanei.

Con medesimo acume, Farinella ${ }^{97}$ rileva un aspetto ideologico fondamentale, insito nel recupero e nella esibizione del Laocoonte, e non solo, da parte di Giulio II:

«il rapporto tematico [...] tra il Laocoonte (con le sue palesi allusioni al mito della fondazione di Roma), l'Apollo (il protettore delle arti e delle lettere che Augusto aveva eletto a proprio nume tutelare) e la Venere (la progenitrice divina della gens Iulia e di quel Giulio Cesare in cui il papa amava rispecchiarsi) doveva suonare, a chi sapesse intendere le allusioni alla storia contemporanea celate nei miti classici, come un'implicita esaltazione del pontificato di Giulio II e della sua politica di rifondazione di Roma, capace di avviare una nuova età dell'oro».

La ripresa del volto del sacerdote troiano per il ritratto di Omero si comprende e si motiva alla luce della renaissance perseguita dal Papa in ambito politico e culturale. Per la sua attuazione le scoperte di antichità e il loro valore simbolico erano fondamentali, tanto più nel caso di una immagine-chiave per la fondazione della Roma renascens Iuliana quale era il Laocoonte, opera che, inoltre, dal 1506 incarnò agli occhi del Papa e di Roma le parole degli auctores antiqui, di Plinio il Vecchio e soprattutto di Virgilio e che ricevette attenzione, non senza motivo, da parte di uno degli umanisti più quotati nei cenacoli intellettuali di Roma, compreso quello iuliano: Jacopo Sadoleto. Questo poeta e letterato inneggiò alla scoperta con il poemetto De Laocoontis statua (1506), molto diffuso e apprezzato, e la sua verosimile presenza nel Parnaso, nel personaggio barbato ritratto accanto alla Musa Urania, sul cui significato ha ben argomentato Alberto Casadei, ${ }^{98}$ darebbe ulteriore forza ideologica all'opzione iconografica del volto di Laocoonte per il ritratto di Omero.

Poco prima della scoperta dell'opera dei summi artifices rodiesi ${ }^{99}$ nel 1505 , fu pubblicata la prima edizione a stampa, presso Aldo Manuzio, dei Posthomerica o Paralipomeni a Omero di Quinto Smirneo (o Quinto Calabro), detto dai primi commentatori rinascimentali

\footnotetext{
95 Maffei 1999, 87-88.

96 Plin. nat. 36,37: sicuti in Laocoonte qui est in Titi imperatoris domo, opus omnibus et picturae et statuariae artis praeferendum. Ex uno lapide eum ac liberos draconumque mirabiles nexus de consilii sententia fecere summi artifices: Hagesander et Polydorus et Athenodorus Rhodii.

97 Farinella 2014, 350-351.

98 Casadei, in Casadei/Farinella 2017, 66-67, 69, spiegando che la «rappresentazione» di Sadoleto «corrisponderebbe a una sorta di metonimia dell'intero ambiente vicino alla corte di Giulio, che da tempo andava affermando la propria autonomia rispetto all'odiato predecessore», Alessandro VI, «e chiedeva che venissero sottolineati i suoi meriti nel restauro della grande arte antica, magari attraverso il sostegno della poesia latina moderna» (ibidem, 67).

99 Plin. nat. $36,37$.
} 


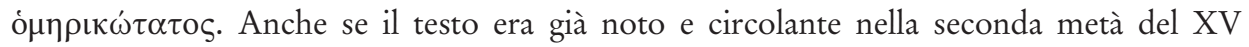
secolo, soprattutto grazie a Costantino Lascaris e ad Angelo Poliziano, ${ }^{100}$ l'edizione manuziana determinò una notevole diffusione del seguito dell' Iliade ${ }^{101}$ presso poeti e letterati del XVI secolo.

Con i Posthomerica Smirneo intese ricollegarsi ai due poeti antonomastici del ciclo troiano, Omero e Virgilio, introducendo delle varianti, una delle quali, per noi molto significativa, riguarda Laocoonte:

[...] buia, intorno alla sua testa / la notte piombò, un acuto dolore colpì le sue palpebre / e sotto le irsute sopracciglia gli occhi furono sconvolti: / le pupille, trapassate da dolorose fitte, / dal profondo erano tormentate; si stravolgevano gli occhi / punti dall'interno, un'acuta sofferenza arrivava / fino alle meningi e nella profondità del cervello. / Allora apparivano rigati di molto sangue i suoi / occhi, come afflitti da incurabile glaucoma; / trasudavano molto come quando da dura roccia / sgorga dai monti acqua mista a neve. / Sembrava essere impazzito e vedeva tutto doppio, / grandi lamenti emettendo. Ma ancora ai Troiani dava ordini / e non si curava della sofferenza. Infine la nobile luce gli tolse / la dea divina: sotto le palpebre le pupille rimangono bianche / esaurito il fiotto del sangue funesto. ${ }^{102}$

Anche attraverso le parole di Quinto Smirneo è chiaro che, all'inizio del XVI secolo, il volto più legittimato a essere il modello per Omero non poteva che essere quello di Laocoonte. Il sacerdote-veggente, pur assente nell' Iliade, era diventato, grazie a Virgilio e alla rilettura dei suoi versi da parte di Giulio II e del suo cenacolo di letterati e artisti, una delle figure simbolo dell'epos troiano, di cui l'Iliade di Omero era indiscussa pietra miliare e di cui i Posthomerica di Smirneo costituivano autorevole ripresa e sviluppo; Posthomerica che, come il Laocoonte scultoreo di Agesandro, Atenodoro e Polidoro, erano una delle scoperte del Rinascimento. ${ }^{103}$

Uscendo dalla Stanza e tornando al Cortile del Belvedere e a Laocoonte, non si può non notare che, anche per il giovane, Raffaello ha guardato al gruppo scultoreo: la postura del suo volto è la stessa che i summi artifices attribuirono al figlio alla sinistra del sacerdote, che nel solo padre spera di trovare la possibilità di continuare a vivere, e così nel viso del fanciullo rivolto con stessa accentuata torsione verso Omero, Raffaello fa trapelare, enfatizzandola, l'espressione di rapimento per la parola del Poeta, dalla quale prenderanno vita le parole degli altri poeti.

Con la scelta del gruppo di Laocoonte per tradurre visivamente Omero e il giovane che trascrive i suoi versi, Raffaello esprime da un lato di condividere la visione ideologica e culturale di Giulio II (e Inghirami), dall'altro afferma in modo eclatante il proprio classicismo, ${ }^{104}$ proclamandosi erede di quei summi artifices, geniali creatori del Laocoonte, di cui Plinio aveva conservato memoria.

100 Megna 2014, 121-161; Nicosia 2014.

101 Riprendo il titolo di Lelli 2013, cui rinvio per l'Introduzione, in particolare per i paragrafi 1, 6, 7.

102 Q. Smyrn. 12,400-415 (traduzione di Lorenzo Ciolfi in Lelli 2013, 561); 12. 389-399; 416-422; 444-499, in particolare 485, 497, 499 con altre menzioni della cecità di Laocoonte.

${ }^{103}$ I Posthomerica furono scoperti dal citato cardinale Bessarione nel monastero di Casole, vicino Otranto, intorno alla metà del XV secolo.

${ }^{104}$ Mariani 1959, 162-172; Becatti 1969, 3-15. 
Omero e il giovane rapsodo, ${ }^{105}$ insieme ad altre figure protagoniste del Parnaso e alla stessa impostazione dell'affresco, avvalorano la vastità e l'eterogenità dell'archivio iconografico e culturale di Raffaello, la sua straordinaria capacità di assorbire, sperimentare, risemantizzare.

Nel monocromo al di sotto della parte sinistra del Parnaso, in corrispondenza del gruppo con i poeti epici guidati da Omero, Raffaello rappresenta il salvataggio dell'Iliade da parte di Alessandro Magno (Alessandro che fa riporre in un cofano di Dario l'Iliade di Omero), concretamente aiutato da un soldato che depone il testo omerico in un forziere classicheggiante. ${ }^{106}$ Questo episodio era usato nel Rinascimento per indicare il rapporto fra letteratura, arti, armi e per simboleggiare il patrocinio delle manifestazioni artistiche da parte dei sovrani. Inoltre, fa parte di quella leggendaria «venerazione» ${ }^{107}$ di Alessandro per Omero, su cui si era soffermato Petrarca, e che Baldassarre Castiglione, tra i plausibili ideatori della Stanza e molto legato a Raffaello, ${ }^{108}$ aveva ripreso dal poeta aretino nel Cortegiano. ${ }^{109}$

La relazione topografica e semantica con Omero e il giovane scripturus è evidente ed è richiamata anche dall'identico schema del braccio destro di Omero e del Macedone.

Che Omero e l'Iliade, testo "viatico di virtù bellica» ${ }^{110} \mathrm{e}$ "specchio verissimo della vita umana», ${ }^{111}$ avessero una positio princeps per Giulio II, i suoi letterari e artisti è incontrovertibile. Altrettanto chiara è la funzione essenziale di trasmettere e conservare la parola alata, svolta dal giovane che sta per mettere per iscritto la poesia del poetarum princeps.

Di quel giovane e della sua funzione non resta memoria nella più icastica apoteosi di Omero ispirata al Parnaso di Raffaello, l'Homère déifié di Jean-Auguste-Dominique Ingres (1827). ${ }^{112}$ In questo dipinto, Omero è poeta divino, icona ieratica e solenne seduta difronte a un tempio a lui intitolato, come si legge nell'epigrafe onomastica che campeggia al centro del fregio. Ai suoi piedi siedono le personificazioni dell'Iliade e dell'Odissea, indicate da iscrizioni in greco, e spiriti magni dell'antichità convergono verso il Poeta da onorare fra gli dèi o da ritenere, comunque, un dio, parafrasando l'altra epigrafe sul basamento del trono su cui siede Omero. Tra i grandi del passato che hanno il sacro diritto di appartenere alla corte del divino Omero, Ingres non dimentica Raffaello: dà al pittore del Parnaso vaticano una posizione di rilievo e un compagno d'eccezione, il pittore Apelle, che tenendolo per mano, afferma e manifesta il legame indissolubile e imperituro di Raffaello con l'antico.

Agosti, B., La funzione della Stanza della Segnatura al tempo di Giulio II nella storiografia artistica del Novecento, in: N. Bock, I. Foletti, M. Tomasi (a cura di), Survivals, Revivals, rinascenze. Studi in onore di Serena Romano, Roma 2017, 523-530.

Battaglia Ricci, L., L’Omero di Boccaccio, in: A. M. Cabrini, A. D’Agostino, Boccaccio: gli antichi e i moderni, Milano 2018, 7-45.

105 Fornaro 2018, 1025-1042; Fornaro/Viccei 2020, 32-33.

106 Cannata Salomone 1997, 76-78; Farinella 2014, 360, diversamente Moormann 2004, 138; Casadei/ Farinella 2017, 68-69. Nell'altro monocromo al di sotto della parte destra del Parnaso è significativamente raffigurato Augusto che impedisce agli esecutori testamentari di Virgilio di distruggere l'Eneide.

107 «Ché, come sapete, Alessandro ebbe in tanta venerazione Omero, che la Iliade sempre si teneva a capo del letto", come si legge nel Cortegiano $(1,43)$ di Baldassarre Castiglione.

108 Di consolidato e "profondo legame affettivo e intellettuale» tra Raffaello e Castiglione, con riferimenti ad alcuni testi emblematici e al famoso Ritratto di Baldassarre Castiglione, opera dell’Urbinate, Farinella 2016.

109 Villa 2010, 211-212, 218-219.

110 Plut. Alex. 8,2; Villa 2010, 219, n. 28.

111 Citazione dal Profugiorum ab aerumna libri, III, 2 di Leon Battista Alberti; Villa 2010, 219, n. 28.

112 https://www.louvre.fr/oeuvre-notices/homere-deifie. 
Becatti, G., Il classico in Raffaello, Accademia Nazionale dei Lincei 132, 1969, 3-15.

Bejor, G. (a cura di), Il Laocoonte dei Musei Vaticani. 500 anni dalla scoperta, Atti del Convegno (Milano, 16 gennaio 2006), Milano 2007.

Bellori, L., Della vita e delle pitture di Raffaello da Urbino per Vasari, Bellori e Missirini, Milano, per Niccolò Bettoni, 1825.

Bellosi, L. / A. Rossi, Le vite de’ più eccellenti pittori, scultori ed architetti di Giorgio Vasari, Torino 1986.

Benedetti, S., Inghirami, Tommaso, detto Fedra, Dizionario Biografico degli Italiani, vol. 62, 2004. URL: http://www.treccani.it/enciclopedia/inghirami-tommaso-detto-fedra_(Dizionario-Biografico).

Bertuzzi, A., Ottaviano Ubaldini Della Carda e l'allestimento della biblioteca di Federico da Montefeltro, Theory and Criticism of Literature and Arts 3, 2018, 146-169.

Bianco, C., L’Accademia del Bessarione tra Roma e Urbino, in: G. Cerboni Baiardi, G. Chittolini, P. Floriani (a cura di), Federico di Montefeltro: lo stato, le arti, la cultura, Roma 1986, III, 61-79.

Bianco, C., Da Bisanzio a Roma. Studi sul cardinale Bessarione, Roma 1999.

Bianco, C., La presenza degli Umanisti ad Urbino nella seconda metà del Quattrocento, in: F. P. Fiore (a cura di), Francesco di Giorgio alla corte di Federico da Montefeltro, Atti del Convegno Internazionale di Studi (Urbino, 11-13 ottobre 2001), Firenze 2004, $127-145$.

Bolzoni, L., Il lettore creativo. Percorsi cinquecenteschi fra memoria, gioco, scrittura, Napoli 2012.

Bortoletti, F., Revivescenze del Parnaso. Presenza e ricezione del cantor, in: F. Bortoletti (a cura di), L'attore del Parnaso. Profili di attori-musici e drammaturgie d'occasione, Milano Udine 2012, 13-119.

Butler, K. E., La Cronaca rimata di Giovanni Santi e Raffaello, in: L. Mochi Onori (a cura di), Raffaello e Urbino. La formazione giovanile e i rapporti con la città natale, Catalogo della Mostra (Urbino, 4 aprile-12 luglio 2009), Milano 2009, 38-43.

Cannata, N., / M. Signorini, "Per trionfar o Cesare o poeta»: la corona d'alloro e le insegne del poeta moderno, in: P. Canettieri, A. Punzi (a cura di), Dai pochi ai molti, Studi in onore di Roberto Antonelli, Roma 2014, 439-473.

Cannata Salomone, N., A dispetto della morte: il sospiro di Alessandro e la memoria della poesia. Una prima ricognizione delle fonti, in: Z. G. Barański, L. Pertile (edd.), In amicizia. Essays in honour of Giulio Lepschy, The italianist, Special supplement 17, 1997, 56-82.

Cannata Salamone, N., "Evidentia in narratione»: filologia e storia nell'iconografia del Cupido di Michelangelo e della Stanza della Segnatura, in: L. Miglio, P. Supino Martini (a cura di), Segni per Armando Petrucci, Roma 2002, 35-59.

Casadei, A., / V. Farinella, Il Parnaso di Raffaello: criptoritratti di poeti moderni e l'ideologia pontificia, Ricerche di Storia dell'arte 40, 2017, 59-72.

Casali, S., Apollo e Marsia nel proemio del Paradiso, in: C. Cattermole, M. Ciccuto (a cura di), Miti, figure, metamorfosi: l'Ovidio di Dante, Firenze 2019, 25-47.

Cavallaro, A., Il luogo delle Muse di Leone X: la villa della Magliana e le sue decorazioniin: F. Cantatore, C. Casetti Brach, A. Esposito, F. Frova, D. Gallavotti Cavallero, P. Piacentini, F. Piperno, C. Ranieri (a cura di), Leone X. Finanza, mecenatismo, cultura, Atti del Convegno Internazionale (Roma, 2-4 novembre 2015), Roma, 2016, 477-500.

Chapman, H. / T. Henry/ C. Plazzotta (a cura di), Raffaello. Da Urbino a Roma, Catalogo della Mostra (London, National Gallery, 20 ottobre 2004-16 gennaio 2005), Milano 2004 (ed. or. London).

Cieri Via, C. (a cura di), Wolfgang Liebenwein. Studiolo. Storia e tipologia di uno spazio culturale, Modena 2005. 
Clements, R. J., Iconography on the Nature and Inspiration of Poetry in Renaissance Emblem Literature, PMLA 70, 4, 1955, 781-804.

Clough, C. H., Il tempietto delle Muse e Giovanni Santi, in: R. Varese (a cura di), 1999, Giovanni Santi, Atti del Convegno Internazionale di Studi (Urbino, 17-19 marzo 1995), Milano 1999, 63-70.

Davoli, Z. / C. Panizzi, Raffaello e l'incisione europea dal Cinquecento all'Ottocento, Catalogo della Mostra (Reggio Emilia, Biblioteca Panizzi, 8 febbraio-5 aprile 2020), Reggio Emilia 2020.

De' Spagnolis, M., L'antologia omerica di Sperlonga. Storia di una grande scoperta archeologica, Rende 2017.

De Vecchi, P. L., Difficultà/facilità e sprezzatura nell'opera di Raffaello, in: P. Nitti, M. Restellini, C. Strinati (a cura di), Raffaello. Grazia e bellezza, Catalogo della Mostra (Paris Musée du Luxembourg, 10 ottobre 2001-27 gennaio 2002), Ginevra-Milano 2001, 29-38.

Di Teodoro, F. P., / V. Farinella, Santi (Sanzio), Raffaello, in Dizionario Biografico degli italiani, XC, Roma 2017, 418-435.

Emiliani, A. / M. Scolaro, Raffaello. La Stanza della Segnatura, Milano 2002.

Farinella, V., Raffaello, Milano 2004.

Farinella, V., Alfonso I d'Este. Le immagini e il potere: da Ercole de' Roberti a Michelangelo, Milano 2014.

Farinella, V., Avventure e metamorfosi di un genio: Raffaello da Urbino a Roma, in: I Classici della Pittura, 2016. URL:http://www.treccani.it/enciclopedia/avventure-e-metamorfosi-di-un-genio-raffaello-da-urbino-a-roma_(altro)/.

Feo, M., Le <due redazioni> della Collatio laureationis, Quaderni petrarcheschi 7, 1990, 186-203.

Ferino Pagden, S. (a cura di), Disegni umbri del Rinascimento da Perugino a Raffaello, Firenze 1982.

Fornaro, S., Il giovane rapsodo nella Stanza della Segnatura di Raffaello, in: S. Bigliazzi, F. Lupi, G. Ugolini, $\Sigma v v \alpha \gamma \omega v i \zeta \varepsilon \sigma \theta \alpha \iota$ Studies in Honour of Guido Avezzù, Verona 2018, 1025-1042.

Fornaro, S., / R. Viccei, Immaginare Omero nel Rinascimento, in: V. Prosperi, F. Ciccolella (a cura di), La fortuna di Omero nel Rinascimento tra Bisanzio e l'Occidente, Alessandria 2020, 17-41.

Foti, G., La lettera di Petrarca a Omero, Parma 2007.

Frommel, C. L., Il palazzo Ducale di Urbino e la nascita della residenza principesca del Rinascimento, in: F. P. Fiore, Francesco di Giorgio alla corte di Federico da Montefeltro, Atti del Convegno Internazionale di Studi (Urbino, 11-13 ottobre 2001), Firenze 2004, 167-196.

Frommel, C. L., Raffaello. Le Stanze, Milano 2017.

Galilei, V., Dialogo di Vincentio Galilei nobile fiorentino della musica antica, et della moderna, in Fiorenza, appresso Giorgio Marescotti, 1581.

Geri, L., Il ritorno delle Muse e la via al Parnaso. Metafore della Rinascita tra Dante, Petrarca e Boccaccio, in: B. Alfonzetti, G. Baldassarri, E. Bellini, S. Costa, M. Santagata (a cura di), Per civile conversazione. Con Amedeo Quondam, Roma 2014, I, 617-631.

Guarino, R., Archeologia e spettacolo a Roma nell'età di Giulio II, in: F. Cantatore, M. Chiabò, P. Farenga, M. Gargano, A. Morisi, A. Modigliani, F. Piperno (a cura di), Metafore di un Pontificato. Giulio II (1503-1513). Atti del Convegno (Roma, 2-4 dicembre 2008), Roma 2010, 345-364.

Guccini, G., Prima del Parnaso. Vocalità vs oralità, performance vs scrittura: una poetica in immagini di Raffaello Sanzio, in: F. Bortoletti (a cura di), L'attore del Parnaso. Profili di attori-musici e drammaturgie d'occasione, Milano / Udine 2012, 461-481.

Guidobaldi, N., Il ritorno delle Muse nel Quattrocento, RIdIM/RCMI Newsletter 17, 1, 1992, 15-24. 
Guidobaldi, N., Il Concerto delle Muse nella Città Ideale: Indagini sul Programma Iconografico del Tempietto del Palazzo Ducale di Urbino con un'Ipotesi di Ricostruzione Virtuale (The Concert of the Muses in the Ideal City: Investigations on the Iconographic Program of the Tempietto of the Palazzo Dicale of Urbino with a Virtual Reconstruction Hypothesis), in: A. Baldassarre, T. Marković (edd.), Music Cultures in Sound, Words and Images. Essays in Honor of Zdravko Blažeković, Wien 2018, 479-498.

Gutman, H. B., Zur Ikonologie der Fresken Raffaels in der Stanza della Segnatura, Zeitschrift für Kunstgeschichte 21, 1, 1958, 27-39.

Henry, T., / F. F. Mancini, (a cura di), Gli esordi di Raffaello tra Urbino, Città di Castello e Perugia, Catalogo della Mostra (Città di Castello, Palazzo Vitelli alla Cannoniera, 24 marzo11 giugno 2006), Città di Castello 2006.

Iacobini, A., 1999, Giovanni Santi e Raffaello, padre e figlio: storia di un mito purista, in: R. Varese (a cura di), Giovanni Santi, Atti del Convegno Internazionale di Studi (Urbino, 17-19 marzo 1995), Milano 1999, 198-205.

Joost-Gaugier, C. L., Raphael's Stanza della Segnatura: Meaning and Invention, London 2002.

Kirкнам, V., Le tre corone e l'iconografia di Boccaccio, in: M. Marchiaro, S. Zamponi (a cura di), Boccaccio letterato, Atti del Convegno Internazionale (Firenze - Certaldo, 10-12 ottobre 2013), Firenze 2015, 453-484.

La Rocca, E., Il ritratto e la somiglianza, in: E. La Rocca, C. Parisi Presicce (a cura di), I giorni di Roma. Ritratti. Le tante facce del potere, Catalogo della Mostra (Roma, Musei Capitolini - Palazzo Caffarelli - Palazzo dei Conservatori, 10 marzo-23 ottobre 2011), Roma 2011, 21-29.

Lelli, E. (a cura di), Quinto di Smirne. Il seguito dell'Iliade, Milano 2013.

Maffei, S., La fama di Laocoonte nei testi del Cinquecento, in: S. Settis, Laocoonte, Fama e stile, Roma 1999, 98-230.

Maggi, G. C. (a cura di), La Collatio Laureationis / Francesco Petrarca, Milano 2004.

Macioce, S., Una nota per il tempietto delle Muse nel palazzo Ducale di Urbino, in: R. Varese (a cura di), Giovanni Santi, Atti del Convegno Internazionale di Studi (Urbino, 17-19 marzo 1995), Milano 1999, 71-76.

Marchi, A. (a cura di), Lo Studiolo del Duca. Il ritorno degli uomini illustri alla corte di Urbino, Catalogo della mostra (Urbino, Galleria Nazionale delle Marche, 12 marzo-4 luglio 2015), Milano 2015.

Mariani, V., Raffaello e il mondo classico, Studi Romani 7, 2, 1959, 162-172.

Megna, P., Per la fortuna umanistica di Quinto Smirneo, Medioevo greco 14, 2014, 121-161.

Mercalli, M. / D. Pagliai, Baldassarre Peruzzi. Sala del Fregio, in: L. Cassanelli, S. Rossi (a cura di), I luoghi di Raffaello a Roma, Catalogo della Mostra (Roma, vari luoghi, 12 gennaio-30 marzo 1984), Roma 1983, 32-38.

Merisalo, O., Baldassarre Turini, funzionario e mecenate, in: F. Cantatore, C. Casetti Brach, A. Esposito, F. Frova, D. Gallavotti Cavallero, P. Piacentini, F. Piperno, C. Ranieri (a cura di), Leone X. Finanza, mecenatismo, cultura, Atti del Convegno Internazionale (Roma 2-4 novembre 2015), Roma 2016, 237-245.

Michelini Tocci, L. (a cura di), Giovanni Santi, La vita e le gesta di Federico da Montefeltro duca di Urbino. Poema in terza rima (Codice Vat. Ottob. Lat. 1305), Città del Vaticano 1985, I-II.

Mochi Onori, L. (a cura di), Raffaello e Urbino. La formazione giovanile e i rapporti con la città natale, Catalogo della Mostra (Urbino, Galleria Nazionale delle Marche, 4 aprile12 luglio 2009), Milano 2009.

Moormann, E. M., «The Man who Made the Song Was Blind»: Representations of Homer in Modern Times, I, Pharos 12, 2004, 129-150. 
Moppi, G., Giulio II e la musica, in: F. Cantatore, M. Chiabò, P. Farenga, M. Gargano, A. Morisi, A. Modigliani, F. Piperno (a cura di), Metafore di un Pontificato. Giulio II (15031513). Atti del Convegno (Roma, 2-4 dicembre 2008), Roma 2010, 295-322.

Morelli, A., Il ritratto di musicista nel Cinquecento: tipologie e significati, in: A. Galli, C. Piccinini, M. Rossi (a cura di), Il ritratto nell'Europa del Cinquecento, Atti del Convegno (Firenze, 7-8 novembre 2002), Firenze 2007, 169-191.

Most, G. W., How many Homers?, in: A. Santoni (a cura di), L’Autore multiplo, Pisa 2004, 1-14.

Nicosia, R., Alla scuola di Omero: Costantino Lascaris e la traduzione latina dell'Odissea nel De Aetna di Pietro Bembo, I Tatti Studies in the Italian Renaissance 17, 2, 2014, 303-324.

Parisi Presicce, C., Inventare volti antichi:i ritratti di ricostruzione, in: E. La Rocca, C. Parisi Presicce (a cura di), I giorni di Roma. Ritratti. Le tante facce del potere, Catalogo della Mostra (Roma, Musei Capitolini - Palazzo Caffarelli - Palazzo dei Conservatori, 10 marzo23 ottobre 2011), Roma 2011, 121-136.

Patetta, T., 〈Antico〉 e 〈Pagano〉 nella libera rilettura di Michelangelo, in: L. Rotondi Secchi Tarugi (a cura di), Roma pagana e Roma cristiana nel Rinascimento. Atti del XXIV Convegno Internazionale (Chianciano Terme-Pienza, 19-21 luglio 2012), Firenze 2014, 185-204.

Peruzzi, M., «Lectissima politissimaque volumina» : i fondi Urbinati, in: C. Montuschi (a cura di), La Vaticana nel Seicento (1590-1700). Una biblioteca di biblioteche, Città del Vaticano 2014, 337-394

Pontani, F., A Byzantine Portrait of Homer, Journal of the Warburg and Courtauld Institutes 68, 2005, 1-26.

Pope-Hennessy, J., Paradiso: The Illuminations to Dante's Divine Comedy by Giovanni di Paolo, London 1993.

Puddu, R., Lettere e armi: il ritratto del guerriero tra Quattro e Cinquecento, in: G. Cerboni Baiardi, G. Chittolini, P. Floriani (a cura di), Federico di Montefeltro: lo stato, le arti, la cultura, Roma 1986, I, 487-512.

Rijser, D., Raphael's Poetics: Art and Poetry in High Renaissance Rome, Amsterdam 2012.

Rinaldi, S. / C. Falcucci, Raffaello lettore di Plinio e la tecnica del chiaroscuro, in: F. Cantatore, M. Chiabò, P. Farenga, M. Gargano, A. Morisi, A. Modigliani, F. Piperno (a cura di), Metafore di un Pontificato. Giulio II (1503-1513). Atti del Convegno (Roma, 2-4 dicembre 2008), Roma 2010, 387-402.

Rodeschini, M. C., / E. Daffra / G. Di Pietrantonio (a cura di), Raffaello e l'eco del mito, Catalogo della Mostra (Bergamo, Galleria d'Arte Moderna e Contemporanea, 27 gennaio 2018-6 maggio 2018), Milano e Firenze 2018.

Rotondi Terminiello, G., Il palazzo di Federico, un'abitazione regale del Rinascimento, in: A. Bruschettini (a cura di), Il Montefeltro e l'Oriente islamico. Urbino 1430-1550. Il Palazzo Ducale tra Occidente e Oriente, Catalogo della Mostra (Urbino, Galleria Nazionale delle Marche Palazzo Ducale, 23 giugno-30 settembre 2018), Genova 2018, 15-18.

Scrivano, R., Cultura letteraria di Raffaello, Studi Romani 31, 3, 1983, 265-285.

SetTis, S., Laocoonte. Fama e stile, Roma 1999.

Shearman, J., Raphael in early modern sources (1483-1602), New Haven-London 2003, I-II. Sток, F., Aeneas redivivus: Piccolomini and Virgil, PAN 7, 2018, 163-176.

Strinati, C., Biografia di Raffaello dal punto di vista del ritratto, in: P. Nitti, M. Restellini, C. Strinati (a cura di), Raffaello. Grazia e bellezza, Catalogo della Mostra (Parigi, Musée du Luxembourg, 10 ottobre 2001-27 gennaio 2002), Ginevra-Milano 2001, 13-27.

Talvacchia, B., When Marcantonio met Raphael, in: M. Israëls, L. A. Waldman (edd.), Renaissance Studies in Honor of Joseph Connors, Florence-Milan 2013, I, 305-310.

TAylor, P., Julius II and the Stanza della Segnatura, Journal of the Warburg and Courtauld Institutes 72, 2009, 103-141. 
Terraroli, V. (a cura di), Raffaello. Lettera a papa Leone X, Milano 2020.

Turner, J. G., Raphael as a Poet, Source 32, 2, 2013, 6-11.

Unglaub, J., Bernardo Accolti, Raphael’s «Parnassus` and a New Portrait by Andrea Del Sarto, Burlington Magazine 149/1246, 2007, 14-22.

Valazzi, M. R., Gli Uomini Illustri dello Studiolo di Federico da Montefeltro. Gli studi. La mostra, in: A. Marchi (a cura di), Lo Studiolo del Duca. Il ritorno degli uomini illustri alla corte di Urbino, Catalogo della Mostra (Urbino, Galleria Nazionale delle Marche, 12 marzo-4 luglio 2015), Milano 2015, 13-17.

Valazzi, M. R. (a cura di), Giovanni Santi. «Da poi ... me dette alla mirabil arte de pictura», Catalogo della Mostra (Urbino, Galleria Nazionale delle Marche, Palazzo Ducale, 29 novembre 2018-17 marzo 2019), Milano 2018.

Varese, R., La Cronaca rimata di Giovanni Santi: proposte di lettura, Notizie da Palazzo Albani $17,2,1988,26-48$.

VArese, R., Giovanni Santi, Fiesole 1994.

Varese, R. (a cura di), Giovanni Santi, Atti del Convegno Internazionale di Studi (Urbino, 17-19 marzo 1995), Milano 1999.

VArese, R., Giovanni Santi teorico e ideologo, in: M. R. Valazzi (a cura di), Giovanni Santi. «Da poi ... me dette alla mirabil arte de pictura», Catalogo della Mostra (Urbino, Galleria Nazionale delle Marche, Palazzo Ducale, 29 novembre 2018-17 marzo 2019), Milano 2018, 75-79.

Vendruscolo, F., Nuove ipotesi sul ritratto riscoperto nel Toledano autografo di Boccaccio, Archivum Mentis 4, 2015, 153-161.

Venturi, A., Il gruppo del Laocoonte e Raffaello, Archivio Storico dell'arte II, 1889, 97-112.

Villa, M., Plutarco e Castiglione: il personaggio di Alessandro Magno, in: M. Gioseffi (a cura di), Uso, riuso e abuso dei testi classici, Milano 2010, 209-232.

Watson, P. F., On a Window on Parnassus, Artibus et Historiae 16, 1987, 127-148.

Watson, P. F., To Paint Poetry: Raphael on Parnassus, in: M. C. Horowitz, A. J. Cruz, W. A. Furman (edd.), Renaissance Rereadings: Intertext and Context, Urbana 1988, 113-141.

Wedepohl, C., In den glänzenden Reichen des ewigen Himmels: Cappella del Perdono und Tempietto delle Muse im Herzogpalast von Urbino, München 2009.

Wilkins, E. H., The Coronation of Petrarch, Speculum 18, 1943, 155-197.

Wilson, B., Singing to the Lyre in Renaissance Italy. Memory, Performance, and Oral Poetry, New York 2020.

Winner, M., / Andreae, B., / Pietrangeli, C., (Hrgg.), Il cortile delle Statue. Der Statuenhof des Belvedere im Vatikan, Mainz 1998.

Winternitz, E., Archeologia musicale del Rinascimento nel Parnaso di Raffaello, Rendiconti della Pontificia Accademia Romana di Archeologia 27, 1952-54, 359-388.

Zanker, P., La maschera di Socrate. L'immagine dell'intellettuale nell'arte antica, Torino 2009 (trad. it.).

Sono state visitate le seguenti fonti Internet il 1.7. 2020.

https://arcade.nyarc.org/record=b1109575 S7 https://blog.naver.com/carandini

https://blog.naver.com/carandini/40190963900

http://collection.mam.org/details.php?id=14910

https://digitalcollections.frick.org/digico/\#/details/bibRecordNumber/b11095751/Photoarchive

http://irds-project.org/doc/129/

http://irds-project.org/doc/1417/

http://irds-project.org/doc/1437/

http://irds-project.org/doc/1947/

http://irds-project.org/doc/2548/ 
http://irds-project.org/doc/1952/

http://irds-project.org/doc/2555/

http://irds-project.org/doc/2559/

http://irds-project.org/doc/2560/

http://irds-project.org/doc/2561/

http://www.bl.uk/manuscripts/Viewer.aspx?ref=yates_thompson_ms_36_fs001ar

https://www.britishmuseum.org/collection/object/G_1819-0812-1

https://www.deviantart.com/jw-jeong

https://www.louvre.fr/oeuvre-notices/homere-deifie

https://www.museoarcheologiconapoli.it/it/le-collezioni/collezione-farnese-2/ritratti/

http://www.regione.marche.it/Regione-Utile/Cultura/Catalogo-beni-culturali/RicercaCatalogoBeni/ ids/53773/Ritratto-del-cardinale-Bessarione.

https://www.tate.org.uk/art/artworks/lawrence-homer-reciting-his-poems-t01974

https://www.thestar.com.my/lifestyle/culture/2014/08/08/joongwon-jeong-hyperrealist-artist 